\title{
GEOMETRIC ANALYSIS
}

\author{
SHING-TUNG YAU
}

This was a talk I gave in the occasion of the seventieth anniversary of the Chinese Mathematical Society. I dedicated it in memory of my teacher S. S. Chern who passed away half a year ago.

During my graduate study, I was rather free in picking research topics. I [538] worked on fundamental groups of manifolds with non-positive curvature. But in the second year of my study, I started to look into differential equations on manifolds. While Chern did not express much opinions on this part of my research, he started to appreciate it a few years later. In fact, after Chern gave a course on Calabi's works on affine geometry in 1972 in Berkeley, Cheng told me these inspiring lectures. By 1973, Cheng and I started to work on some problems mentioned in his lectures. We did not realize that great geometers Pogorelov, Calabi and Nirenberg were also working on them. We were excited that we solved some of the conjectures of Calabi on improper affine spheres. But soon we found out that Pogorelov [398] published it right before us by different arguments. Nevertheless our ideas are useful to handle other problems in affine geometry and my knowledge about Monge-Ampère equations started to be broadened in these years. Chern was very pleased by my works, especially after I [543] solved the problem of Calabi on Kähler Einstein metric in 1976. I had been in Stanford and Chern proposed to Berkeley Department to hire me. I visited Berkeley in 1977 for a year and gave a course on geometric analysis with emphasis on isometric embedding.

Chern nominated me to give a plenary talk in the International Congress in Helsinki. The talk [544] went well, but my decision not to stay in Berkeley did not quite please him. Nevertheless he recommended me to be a faculty in the Institute for Advanced Study. Before I accepted to be a faculty in the Institute, I organized a special year on geometry in 1979 in the Institute at the invitation of Borel. That was an exciting year because most people in geometric analysis came.

In 1979, I visited China at the invitation of Professor L. K. Hua. I gave a series of talks on the bubbling process of Sacks-Uhlenbeck [413]. I suggested to the Chinese mathematicians to apply similar arguments for Jordan curve bounding two surfaces with same constant mean curvature. I thought it is a good exercise to get into this exciting field of geometric analysis. The problem was indeed picked up by a group of students of Professor Wang [251]. But unfortunately 
it also initiated some ugly fights during the meeting of the sixtieth anniversary of the Chinese Mathematical Society. Professor Wang was forced by one of his students to resign. This event did slow down development of this beautiful subject in China in the past ten years.

In 1980, Chern decided to develop geometric analysis in a large scale. $\mathrm{He}$ initiated a series of international conferences on differential geometry and differential equations to be held each year in China. For the first year, a large group of most distinguished mathematicians were gathering in Beijing to give lectures (see [107]). I lectured on open problems in geometry [546]. It took a much longer time than I expected for Chinese mathematicians to pick up some of these problems.

To his disappointment, Chern's enthusiasm about developing differential equations and differential geometry in China did not stimulate as much activity as he had hoped. Most Chinese mathematicians are trained in analysis, but rather weak in geometry. The goal of geometric analysis to understand geometry is not appreciated. On the other hand, the works of Zhong (see, e.g., [562, 372, 373]) were remarkable. Unfortunately he died about twenty years ago. The major research center on differential geometry come from students of Chern and late Professor Su. Gu [208] did a remarkable pioneering work in which he considered harmonic map based on metrics with different signature. Such maps are called wave maps. Hong (see, e.g., [239, 220]) did some interesting works on isometric embedding of surfaces into $\mathbb{R}^{3}$. In the past five years, the research center in the Chinese University of Hong Kong led by Tam and Zhu did produce first class works related to Hamilton's Ricci flow (see, e.g., [87, 88, 91, 92, 80]).

As a support of Chern's ambition on building up geometric analysis, I will explain my personal view to my Chinese colleagues.

I will consider this article to be successful if it conveys to my readers the excitement of developments in differential geometry which have been taking place during the period during which it has been my good fortune to contribute. I do not claim this covers all respects of the subject. In fact, I have given priority to those works closer to my personal experience, and, as such, it gives insufficient space to aspects of differential geometry in which I have not participated. In spite of its shortcomings, I hope that its readers, particularly those too young to know the origins of geometric analysis will be interested to learn how the field looks to someone who was there. I am grateful to J. Fu, especially for his help tracking down references.

The major contributors can be roughly mentioned in the following periods: 
I. 1972 to 1982: Atiyah, Bott, Singer, Calabi, Nirenberg, Pogorelov, Schoen, Simon, Uhlenbeck, Donaldson, Hamilton, Taubes, Stein, Fefferman, Siu, Caffarelli, Kohn, S. Y. Cheng, Kuranishi, Cheeger, Gromoll, Harvey, Lawson, Gromov, Aubin, Patodi, Hitchin, Guillemin, Melrose, Colin de Verdière, M. Taylor, Bryant, H. Wu, R. Green, Peter Li, Phong, Wolpert, Pitts, Trudinger, Hildebrandt, Kobayashi, Hardt, Spruck, Gerhardt, White, Gulliver, Warner, Kazdan.

II. 1983 to 1992: In 1983, Schoen and I started to give lectures on geometric analysis in the Institute for Advanced Study. Zhong was the major person to take notes of our lectures. The lectures were continued in 1985 in San Diego. During the period of 1985 and 1986, K. C. Chang and W. Y. Ding came to take notes for our lectures. The book Lectures on Differential Geometry was published in Chinese around 1989 [434]. It did have great influence for a generation of Chinese mathematicians to become interested in this subject. At the same time, a large group of my students made contributions to the subject. This includes Treibergs, Parker, Bartnik, Bando, Saper, Stern, Cao, Chow, Shi, Zheng and Tian.

At the same time, Bismut, C. S. Lin, N. Mok, J. Q. Zhong, Jost, Huisken, Jerison, Sarnak, Fukaya, Mabushi, Ilmanen, Croke, Stroock, Price, F. H. Lin, Zelditch, Christodoulou, Klainerman, Moncrief, Terng, Michael Wolf, Anderson, LeBrun, Micallef, Moore, John Lee, A. Chang, Korevaar are making contributions in various directions. One should also mention that in this period important work done by the authors in the first group. For example, Donaldson, Taubes [476] and Uhlenbeck [509, 510] did the spectacular works on Yang-Mills theory on general manifolds which led Donaldson [143] to solve outstanding questions on four manifold topology. Donaldson [144], Uhlenbeck-Yau [512] proved the existence of Hermitian Yang-Mills connection on stable bundles. Schoen [419] solved the Yamabe problem.

III. 1993 to now: Many mathematicians joined the subject. This includes Kronheimer, Mrowka, Demailly, Colding, Minicozzi, T. Tao, Thomas, Zworski, Eliasberg, Toth, Andrews, L. F. Tam, C. Leung, Y. Ruan, W. Ruan, R. Wentworth, Grigorian, Saloff-Coste, J. X. Hong, X. P. Zhu, M. T. Wang, A. K. Liu, K. F. Liu, X. F. Sun, T. J. Li, X. J. Wang, Loftin, Y. Li, H. Bray, J. P. Wang, L. Ni, P. F. Guan, N. Kapouleas, Ozsváth, Szabó, Y. Li. The most important event is of course the first major breakthrough of Hamilton [218] in 1995 on the Ricci flow. I did propose to him in 1982 to use his flow to solve Thurston's conjecture. But only until this paper of Hamilton, it is finally realized that it is feasible to solve the full geometrization program by geometric analysis. (A key step was estimates on parabolic equations initiated by Li-Yau [309] and accomplished by Hamilton for Ricci flow [216, 217].) In 2002, Perelman [389, 390] brought in fresh new ideas to solve important steps that are remained in the program. It is hopeful that the works of Chen-Zhu [91, 92] and Cao-Zhu may finally finish the whole program. In the other direction, we see the important 
development of Seiberg-Witten theory [533]. Taubes [482, 483, 484, 485] was able to prove the remarkable theorem on counting pseudo-holomorphic curves in terms of his invariants. Kronheimer-Mrowka [279] were able to solve the Thom conjecture that holomorphic curves provide the lowest genus surfaces in representing homology in algebraic surfaces. (Ozsváth-Szabó had a symplectic version [387].)

Since the whole development does depend heavily on the past, we start out with historical developments. The followings are samples of work before 1970 which provide fruitful ideas and methods.

- Fermat's principle of calculus of variation (Shortest path in various media).

- Calculus (Newton and Leibniz): Path of bodies governed by law of nature.

- Euler, Lagrange: Foundation for variational principle and the study of partial differential equations. Derivations of equations for fluids and for minimal surfaces.

- Fourier, Hilbert: Decomposition of functions into eigenfunctions, spectral analysis.

- Gauss, Riemann: Concept of intrinsic geometry.

- Riemann, Dirichlet, Hilbert: Solving Dirichlet boundary value problem for harmonic function using variational method.

- Maxwell: Electromagnetism, gauge fields, unification of forces.

- Christoffel, Levi-Civita, Bianchi, Ricci: Calculus on manifolds.

- Riemann, Poincaré, Koebe: Riemann surface uniformation theory, conformal deformation.

- Cartan: Exterior differential system, connections on fiber bundle.

- Einstein, Hilbert: Einstein equation and Hilbert action.

- Dirac: Spinors, Dirac equation, quantum field theory.

- Kähler, Hodge: Kähler metric and Hodge theory.

- Hilbert, Cohn-Vossen, Lewy, Weyl, Hopf, Pogorelov, Effimov, Nirenberg: Global surface theory in three space based on analysis.

- Weistress, Riemann, Lebesgue, Courant, Douglas, Radó, Morrey: Minimal surface theory.

- Gauss, Green, Poincaré, Schauder, Morrey: Potential theory, regularity theory for elliptic equations.

- Weyl, Hodge, Kodaira, de Rham, Bergman, Milgram-Rosenbloom, Atiyah-Singer: de Rham-Hodge theory, integral operators, kernel functions, index theory.

- Pontrjagin, Chern, Allendoerfer-Weil: Global topological invariants defined by curvarure forms. 
- Bochner-Kodaira: Vanishing of cohomology groups based on curvature consideration.

- De Giorgi-Nash-Moser: Regularity theory for higher dimensional elliptic equation and parabolic equation of divergence type.

- Kodaira, Morrey, Hörmander, Kohn, Andreotti-Vesentini: Embedding of complex manifolds, $\bar{\partial}$-Neumann problem.

- Kodaira-Spencer, Newlander-Nirenberg: Deformation of geometric structures.

- Federer-Fleming, Almgren, Allard: Varifolds and minimal varieties in higher dimension.

- Eells-Sampson: Existence of harmonic maps into manifolds with nonpositive curvature.

- Calabi: Affine geometry and conjectures on Kähler Einstein metric.

I will now explain what has been accomplished since the seventies, and I will begin with a more systematic study of interaction between geometry and analysis. The story depends on my personal tastes and may leave out the contribution of many authors.

\section{Construction of functions in geometry}

The following is the basic principle [544]:

Linear or non-linear analysis is developed to understand the underlying geometric or combinational structure. On the process, geometry will provide deeper insight of analysis. An important guideline is that space of special functions defined by the structure of the space can be used to define the structure of this space itself.

Algebraic geometer has defined Zariski topology of an algebraic variety using ring of rational functions.

In differential geometry, one should extract information about the metric and topology of the manifolds from functions defined over it. Naturally, these functions should be defined either by geometric construction or by differential equations given by the underlying structure of the geometry. (Integral equations have not been used extensively as the idea of linking local geometry to global geometry is more related to differential equations.) A natural generalization of functions consists of following: differential forms, spinors, sections of vector bundles.

The dual concepts of differential forms or sections of vector bundles are submanifolds or foliations. From the differential equations that govern them (mostly 
arising from variational principle) we have minimal submanifolds or holomorphic cycles. Naturally the properties of them or the totality of all such objects govern the geometry of the underlying manifold. A very good example is Morse theory on the space of loops on a manifold.

I shall now discuss various methods for constructing functions or tensors of geometric interest.

\section{A. Polynomials from ambient space.}

If the manifold is isometrically embedded into Euclidean space, a natural class of functions are the restrictions of polynomials from Euclidean space. However, isometric embedding in general is not rigid, and so functions constructed in such a way are usually not too useful.

On the other hand, if a manifold is embedded into Euclidean space in a canonical manner and the geometry of this submanifold is defined by some group of linear transformations of the Euclidean space, the polynomials restricted to the submanifold do play important roles. S. Y. Cheng and I $(1974,1975)[102,106]$ did develop several important gradient estimates for these functions in order to control the geometry of such submanifolds.

The first important theorem is a spacelike hypersurface $M$ in the Minkowski space $\mathbb{R}^{n, 1}$. A very important question: Since the metric on $\mathbb{R}^{n, 1}$ is $\sum\left(d x^{i}\right)^{2}-$ $d t^{2}$, the restriction of this metric on $M$ need not be complete even though $M$ may be complete with respect to the Euclidean metric. In order to prove the equivalence of these two concepts for hypersurface where we can control their mean curvature, Cheng and I proved gradient estimate of the function

$$
\langle X, X\rangle=\sum_{i}\left(x^{i}\right)^{2}-t^{2}
$$

restricted on the hypersurface.

By choosing coordinate system, the function $\langle X, X\rangle$ can be assumed to be positive and proper on $M$. For any positive proper function $f$ defined on $M$, if we prove the following gradient estimate

$$
\frac{|\nabla f|}{f} \leq C
$$

where $C$ is independent of $f$, then we can prove the metric on $M$ is complete. This is obtained by integrating the inequality to get

$$
|\log f(x)-\log f(y)| \leq C d(x, y)
$$

so that when $f(y) \rightarrow \infty, d(x, y) \rightarrow \infty$. Once we know the metric is complete, we proved the Bernstein theorem which says that maximal spacelike hypersurface must be linear. Such work was then generalized by Treibergs [506], C. Gerhardt 
[181] and R. Bartnik [28] for hypersurfaces in more general spacetimes. (It is still an important problem to understand the behavior of maximal spacelike hypersurface foliation for general spacetime when we assume the spacetime is evolved by Einstein equation from nonsingular data set.)

Another important example is the study of affine hypersurfaces $M^{n}$ in an affine space $A^{n+1}$. These are the improper affine spheres

$$
\operatorname{det}\left(u_{i j}\right)=1
$$

where $u$ is a convex function or the hyperbolic affine spheres

$$
\operatorname{det}\left(u_{i j}\right)=\left(-\frac{1}{u}\right)^{n+2}
$$

where $u$ is convex and zero on $\partial \Omega$ and $\Omega$ is a convex domain. Note that these equations describe hypersurfaces where the affine normals are either parallel or converge to a point.

For affine geometry, there is an affine invariant metric defined on $M$ which is

$$
\left(\operatorname{det} h_{i j}\right)^{-\frac{1}{n+2}} \sum h_{i j} d x^{i} d x^{j}
$$

where $h_{i j}$ is the second fundamental form of $M$. A fundamental question is whether this metric is complete or not.

If a coordinate system in $A^{n+1}$ is chosen so that the height function is a proper positive function defined on $M$. The gradient estimate of the height function gives a way to prove completeness of the affine metric. Cheng and I [106] did find such an estimate similar to the one given above.

Once completeness of the affine metric is known, it is trivial to prove properties of affine spheres, some of which were conjectured by Calabi. For example we proved that an improper affine sphere is a paraboloid and that every proper convex cone admits a foliation of hyperbolic affine spheres. The statement about improper affine sphere was first proved by Jörgens [252], Calabi [67] and Pogorelov [398]. Conversely, we also proved that every hyperbolic affine sphere is asymptotic to a convex cone. (The estimate of Cheng-Yau was reproduced again by a Chinese mathematician who claimed to prove the result ten years afterwards.) Much more recently, Trudinger and X. J. Wang [508] solved a difficult Bernstein problem for affine minimal surface, thereby settling a conjecture by Chern. They found counterexample for $\operatorname{dim} \geq 10$. These results make it clear that we can learn much more about affine minimal surfaces by examining fourth order elliptic equations.

The same sort of argument, using gradient estimates for some naturally defined function was also used by me to prove that the Kähler Einstein metric constructed by Cheng and myself is complete for any bounded pseudo-convex 
domain [104]. (It appeared in my paper with Mok [371] who proved the converse statement which says that if the Kähler Einstein metric is complete, the domain is pseudo-convex.)

Cheng-Yau's argument is based on maximum principle. Prior to such development, there was deep work due to Bombieri-Ginsti [45] on gradient estimate of minimal graph using ideas of De Giorgi-Nash. Eventually, N. Korevaar [274] found an argument of this work based on maximum principle also.

Basic Principle: To control a metric, find a function that we know well and give a gradient or higher order estimate of this function.

This principle was used by Cheng-Li-Yau [100] in 1982 to give a comparison theorem for heat kernel for minimal submanifolds in Euclidean space, sphere and hyperbolic space. Since any complex submanifold in $\mathbb{C} P^{n}$ can be lifted to a minimal submanifold in $S^{2 n+1}$, the much later work of Li-Tian [304] on complex submanifolds of $\mathbb{C} P^{n}$ is a corollary.

Another very important property of a linear function is that when it is restricted to a minimal hypersurface in a sphere $S^{n+1}$, it is automatically an eigenfunction. When the hypersurface is embedded, I conjectured that the first eigenfunction is linear and the first eigenvalue of the hypersurface is equal to $n$ (see [546]). While this conjecture is not completely solved, the work of ChoiWang [112] gives strong support, and they proved that the first eigenvalue has a lower bound depending only on $n$. Such a result was good enough for ChoiSchoen [110] to prove a compactness result for embedded minimal surfaces in $S^{3}$.

There is another class of functions that are constructed from the ambient space. These are the support functions of a hypersurface which are functions defined on the sphere. The famous Minkowski problem reduces to solving some Monge-Ampère equation for the support function. This was done by Nirenberg [382], Pogorelov[397], Cheng-Yau[103]. The question of prescribed symmetric functions of principal curvatures has been studied by many people: Pogorelov[399], Caffarelli-Nirenberg-Spruck[65], P. F. Guan (see [210, 209]), Bo Guan, Gerhardt[182], etc. The question of isometric embedding of surfaces into three space can also be written in terms of Darboux equation for the support function. For local problem, there are deep works due to C. S. Lin [320, 321], which are followed by Han-Hong-Lin [220]. The global problem for surfaces with negative curvature was studied by Hong [239].

Submanifolds of space forms are called isoparametric if the normal bundle is flat and the principal curvatures are constants along parallel normal fields. These were studied by E. Cartan. Recently there has been extensive work by Terng and Thorbergsson (see Terng's survey [488] and Thorbergsson [493]). Terng [487] also connected hyperbolic spaces embedded in Euclidean space to soliton 
theory. Also, a nice theory of Lax pair and loop groups related to geometry has been developed.

\section{B. Geometric construction of functions}

When manifolds cannot be embedded into linear spaces, there are ways to construct functions adapted to metric structure. Obviously distance function is the first major function to be used. Out of the distance function, we can construct Busemann function in the following way:

$$
\begin{array}{r}
\text { Given a geodesic ray } \gamma:[0, \infty) \rightarrow M \text { so that } \\
\operatorname{distance}\left(\gamma\left(t_{1}\right), \gamma\left(t_{2}\right)\right)=t_{2}-t_{1},
\end{array}
$$

where $\left\|\frac{d \gamma}{d t}\right\|=1$, one defines

$$
B_{\gamma}(x)=\lim _{t \rightarrow \infty}(d(x, \gamma(t))-t) .
$$

This function generalizes the notion of linear function. For hyperbolic spaceform, its level set defines horospheres. For manifolds with positive curvature, it is concave. Cohn-Vossen (for surface) and Gromoll-Meyer [196] used it to prove that a complete noncompact manifold with positive curvature is diffeomorphic to $\mathbb{R}^{n}$.

A very important property of the Busemann function is that it is superharmonic on complete manifolds with nonnegative Ricci curvature in the sense of distribution. This is the key to prove the splitting principle of Cheeger-Gromoll [86]. Various versions of this splitting principle have been important for applications to structure of manifolds. When I [543] proved the Calabi conjecture, the splitting principle was used by me and others to prove the structure theorem for Kähler manifolds with nonnegative first Chern Class. (The argument for the structure theorem is due to Calabi [66] who know how to handle the first Betti number. Kobayashi [267] and Michelsohn [363] wrote up the formal argument and Beauville [33] had a survey article on this development.)

In 1974, I was able to use the Busemann function to estimate volume of complete manifolds with nonnegative Ricci curvature [541]. This work was generalized by Gromov [201] to understand volume of geodesic balls and has been useful for the recent works of Perelman $[389,390]$ on Hamilton's flow.

If we consider $\inf _{\gamma} B_{\gamma}$, where $\gamma$ ranges from all geodesic rays from a point on the manifold, we may be able to obtain a proper exhaustion of the manifold. When $M$ is a complete manifold with finite volume and its curvature is pinched by two negative constants, Siu and I [459] did prove that such function gives a concave exhaustion of the manifold. If the manifold is Kähler, we were able to prove that we can compactify such manifolds by adding a point to each end to form a compact complex variety. 
I also proposed that every complete Kähler manifold with bounded curvature, finite volume and finite topology should be compactificable to be a compact complex variety. I suggested this problem to Mok and Zhong in 1982 who did significant work [373] in this direction. (The compactification by Mok-Zhong is not canonical and it is desirable to find an algebraic geometric analogue of Borel-Baily compactification [20] so that we can study the $L^{2}$-cohomology in terms of the intersection cohomology of the compactification.) Recall that the important conjecture of Zucker on identifying $L^{2}$-cohomology with the intersection cohomology of the Borel-Baily compactification was settled by Saper-Stern [415] and Looijenga [337]. It would be nice to have similar compactification, at least for moduli spaces of various types. Goresky-Harder-MacPherson [190] and Saper [414] has contributed a lot towards this kind of questions.

Besides taking distance function to a point, we can also take distance function to a submanifold. In such case, its Laplacian will involve Ricci curvature of the ambient manifold and the mean curvature of the submanifold. Such functions can be used as barrier for construction of minimal submanifolds. Schoen-Yau [431] was able to use such barrier to apply minimal surface argument to prove that any complete three dimensional manifold with positive Ricci curvature is diffeomorphic to Euclidean space.

If we look at the space of loops in a manifold, we can take the length of each loop and thereby define a natural function on the space of loops. This is a function for which Morse theory found rich application. Starting from the famous works of Poincaré, Birkhoff, Morse, Ljusternik-Shnirel'man, there has been extensive work on proving existence of closed geodesic using Morse theory on the space of loops. Klingenberg and his students developed powerful tools (see [266]). Gromoll-Meyer [197] did important work in which they proved the existence of infinitely many closed geodesics. There was also later work by Ballmann, Ziller, Hingston and Kramer (see, e.g., [24, 227, 278]). When the metric is Finsler, the most recent work of Victor Bangert and Yiming Long [27] shows the existence of two closed geodesics on the two dimensional sphere. (Katok[261] produced an example which shows that two is optimal.) Under the leadership of Chern, David Bao, Z. Shen, X. H. Mo and M. Ji developed Finsler geometry (see, e.g., [29]).

When the manifold has negative curvature, the length function of curves is related to the displacement function defined in the following way:

If $\gamma$ is an element of the fundamental group acting on the universal cover of a complete manifold with non-positive curvature, we consider the function $d(x, \gamma(x))$ : The study of such a function gives rise to properties of compact manifolds with non-positive curvature. For example, in my thesis, I generalized Preissmann theorem to the effect that every solvable subgroup of the fundamental group must be a finite extension of an abelian group which is the 
fundamental group of a totally geodesic flat subtorus [538]. Gromoll-Wolf [198] and Lawson-Yau [284] also proved that if the fundamental group of such manifold has no center and splits as a product, then the manifold splits as a metric product.

When the manifold has bounded curvature, Margulis studied those points where $d(x, \gamma(x))$ is small and proved the famous Margulis lemma which was used extensively by Gromov [199] to study structure of manifolds with non-positive curvature. He [200] also developed a remarkable idea of Morse theory for the distance function (a preliminary version due to K. Grove and K. Shiohama [207]) to compare topology of a geodesic ball to that of a large ball, thereby obtaining a bound on the Betti numbers of compact manifolds with nonnegative sectional curvature. (He can also allow negative curvature when the curvature is bounded below, but then the diameter and lower bound on the curavture enter the estimate.)

The Busemann function also gives a way to detect the "angular structure" at infinity of the manifold. It can be used to construct the Poisson kernel of hyperbolic space form. For simply connected complete manifold with bounded and strongly negative curvature, it is used as a barrier to solve Dirichlet problem for bounded harmonic functions, after they are mollified at infinity. This was achieved by Sullivan [471] and Anderson [4]. Schoen and Anderson [5] also had deep Harnack inequality for bounded harmonic function and identified the Martin boundary of such manifolds. There is also important work due to W. Ballmann [21] on the Dirichlet problem for manifolds of nonpositive curvature.

The Martin boundary was also studied by L. Ji and MacPherson [250] for the compactification of various symmetric spaces. For products of manifolds with negative curvature, there is also nice work of A. Freire [170] about the Martin boundary. For rank one complete manifolds with nonpositive curvature, there is work due to Ballman-Ledrappier [22] and Cao-Fan-Ledrappier [75]. This work should be related to the L. K. Hua operator on symmetric spaces with higher rank. Hua found that bounded harmonic functions satisfy extra equations that come from Hua operator. See [242].

\section{Functions defined by differential equations}

The most important differential operator for a manifold is the Laplacian. Its spectral resolution gives rise to eigenfunctions. Harmonic functions are therefore the simplest functions that play important roles in geometry.

If the manifold is compact, the maximum principle shows that harmonic functions are constant. However, when we try to understand singularities of compact manifolds, we may create noncompact manifolds by scaling and blowing 
up processes, at which point harmonic functions can play an important role.

The first important question about harmonic functions on a complete manifold is the Liouville theorem. I started my research on analysis by understanding the right formulation of Liouville theorem. In 1971, I thought that it is natural to prove that for complete manifolds with non-negative Ricci curvature, there is no nontrivial harmonic function [539]. I also thought that in the opposite case, when a complete manifold has strongly negative curvature and is simply connected, one should be able to solve Dirichlet problem for bounded harmonic functions.

The gradient estimates [539] that I derived for positive harmonic function come from a suitable interpretation of Schwarz lemma in complex analysis. In fact, I generalized the Ahlfors Schwarz lemma before I understood how to work out the gradient estimates for harmonic functions. The generalized Schwarz lemma [545] says that holomorphic maps, from a complete Kähler manifold with Ricci curvature bounded from below to a Hermitian manifold with holomorphic bisectional curvature bounded from above by a negative constant, are distance decreasing with constants depending only on the bound on the curvature. This generalization has since found many applications such as the study of the geometry of moduli spaces by Liu-Sun-Yau [333, 334]

The gradient estimate that I found can be generalized to cover eigenfunctions and Peter Li [301] was the first one to apply it to find estimates for eigenvalues for manifolds with positive Ricci curvature. (If the Ricci curvature has a positive lower bound, this was due to Lichnerowicz.) Li-Yau [307] then solved the problem of estimating eigenvalues of manifolds in terms of their diameter and the lower bound on their Ricci curvature. Li-Yau conjectured the sharp constant for their estimates, and Zhong-Yang [562] were able to prove this conjecture by sharpening Li and Yau's arguments. A probabilistic argument was developed by Chen and Wang [94] to derive these inequalities. The precise upper bound for the eigenvalue was first obtained by S. Y. Cheng [97]. Cheng's theorem gives a very good demonstration of how the analysis of functions provides information about geometry. As a corollary of his theorem, he proved that if a compact manifold $M^{n}$ has Ricci curvature $\geq n-1$ and the diameter is equal to $\pi$, then the manifold is isometric to the sphere. He used a lower estimate for eigenvalues due to Lichnerowicz and Obata. Colding [123] was able to use functions with properties close to those of the first eigenfunction to prove a pinching theorem which states that: When Ricci curvature is bounded below by $n-1$ and volume is close to that of the unit sphere, the manifold is diffeomorphic to the sphere. There is extensive work by Colding-Cheeger $[83,84,85]$ and Perelman (see, e.g., [61]) devoted to understanding of Gromov's theory of Hausdorff convergence for manifolds. The tools they used include the comparison theorem, splitting theorem of Cheeger and Gromoll, and the ideas introduced earlier by Colding. 
The classical Liouville theorem has a natural generalization: Polynomial growth harmonic functions are in fact polynomials. Motivated by this fact and several complex variables, I asked whether the space of polynomial growth harmonic functions with a fixed growth rate is finite dimension with upper bound depending only on the growth rate [548]. This was proved by Colding-Minicozzi [124] and generalized by Peter Li [302]. (Functions can be replaced by sections of bundles). In a beautiful series of papers (see, e.g., [305, 306]), P. Li and J. $\mathrm{P}$. Wang studied the space of harmonic functions in relation to the geometry of manifolds. In the case when harmonic functions are holomorphic, they form a ring. I am curious about the structure of this ring. In particular, When is it finitely generated?

Most of these works can be generalized to those manifolds where Sobolev and Poincaré inequalities hold. (It should be noted that Aubin [17, 19] and Talenti [473] did find best constant for various Sobolev inequalities on Euclidean space.) These inequalities are all related to isoperimetric inequalities. The first important work in this direction was due to Cheeger [81] who pointed out the importance of isoperimetric constant for estimates of eigenvalues. C. Croke [134] was able to follow my work [540] on Poincaré inequalities to prove Sobolev inequality depending only on volume, diameter and the lower bound of Ricci curvature. Arguments of John Nash was then used by Cheng-Li-Yau [99] to give estimates of heat kernel and its higher derivatives. In this paper, an estimate of injectivity radius was derived and this estimate turn out to play a role in Hamilton's theory of Ricci flow. D. Stroock (see [380]) was able to use his methods from Malliavin's calculus to give remarkable estimates for heat kernel at pair of points where one point is at the cut locus of another point.

The estimate of heat kernel was later generalized by Saloff-Coste and Grigor'yan to complete manifolds with polynomial volume growth and volume doubling properties. Since these are quasi-isometric invariants, their analysis can be applied to graphs or discrete groups. See Grigor'yan's survey [193] and SaloffCoste's survey [417].

On the other hand, the original gradient estimate that I derived is a pointwise inequality that is much more adaptable to nonlinear theory. Peter Li and I [309] were able to find a parabolic version of it in 1984. We observed its significance for estimates on the heat kernel and its relation to variational principle for paths in spacetime. Such ideas turn out to provide fundamental estimates which play a crucial role in the analysis of Hamilton's Ricci flow [216, 217].

Isoperimetric inequality is a beautiful subject. It has a long history. Besides its relation to eigenvalues, it reviews deep structure of manifolds. The best constant for the inequality is important. Pólya-Szegö [400], G. Faber (1923), E. Krahn (1925), P. Lévy (1951) made fundamental contributions. Gromov generalized the idea of Lévy to obtain good estimate of Cheeger's constant (see 
[203]). C. Croke [135] and Cao-Escobar [74] have worked on domains in a simply connected manifold with non-positive curvature. It is assumed that the inequality holds for any minimal subvariety in Euclidean space. But it is not known to be true for the best constant. Li-Schoen-Yau [303] did some work in the case of surfaces, and E. Lutwak, Deane Yang and G. Y. Zhang did some beautiful work in the affine geometry case (see, e.g., [343, 344]).

A very precise estimate of eigenvalues of Laplacian has been important in many areas of mathematics. For example, the idea of Szegö [472]-Hersch [226] on upper bound of first eigenvalue was generalized by me to higher genus in joint works with P. Yang [537] and P. Li [308]. I [551] applied it to prove that a Riemann surface defined by an arithmetric group must have a relative high degree when it is branched over the sphere. In a beautiful article, N. Korevaar [275] gave an upper bound, depending only on genus and $n$, for the $n$th eigenvalue $\lambda_{n}$ of Riemann surface. His result settled a conjecture of mine (see [546]). Grigor'yan, Netrusov and I [194] were able to simplify his proof and gave applications to bounding the index of minimal surfaces. There are also beautiful works by P. Sarnak (see, e.g., [416, 249]) on understanding eigenfunctions for such Riemann surfaces. He pointed out their relevance to number theory and showed that the estimate of the maximum norm of $n$-th eigenfunction on arithmetic surface has significant interest in number theory.

There are many important properties of eigenfunctions that were studied in the seventies. For example, Cheng [98] found a beautiful estimate of multiplicities of eigenvalues based only on genus. The idea was used by Colin de Verdière [132] to embedded graphes into $\mathbb{R}^{3}$ when they satisfy nice combinatorial properties. The connectivity and the topology of nodal domains are very interesting questions. Melas [359] did prove that for convex planar domain, the nodal line of second eigenfunctions must intersect the boundary in exactly two points. Very little is known about the number of nodal domains except the famous theorem of Courant that the number of nodal domain of $m$-th eigenfunction is less than $m$.

There are many other ideas in geometric analysis that can be discretized and applied to graph theory. This is especially true for the theory of spectrum of graphs. Some of these were carried out by F.Chung, Grigor'yan and myself (see the reference of Chung's survey [121]). But the results in [121] are far away from being exhaustive. On the other hand, Margulis [347], Lubotzky-Phillips-Sarnak [340] were able to make use of discrete group and number theory to construct expanding graphes. Methods to construct and classify these expanding graphs are important for application in computer science. It is also important to see how to give a good decomposition of any graph using spectral method.

There are several important questions related to nodal sets and the number of critical points of eigenfunctions. I made a conjecture (see [546]) about the 
area of nodal sets, and significant progress toward its resolution was made by Donnelly-Fefferman [153], Dong [152] and F. H. Lin [323]. The number of critical points of eigenfuction is difficult to determine. I [552] managed to prove existence of an critical point near the nodal set. Nadirashvili has proved several nice results concerning eigenvalues and their eigenfunctions. He and his coauthors [238] were the first to show that the critical point sets of solutions to eigenfunctions in $n$-dimensional manifold have finite $\mathrm{H}^{n-2}$-Hausdorff measure. Afterwards, Han-Hardt-Lin [219] gave an explicit estimate.

When there is potential $V$, the eigenvalues of $-\Delta+V$ are also important. I made an attempt to understand the gap $\lambda_{2}-\lambda_{1}$ with Singer, Wong, and stephen Yau [450]. I [553] observed how this gap depends on the lower eigenvalue of the Hessian of $-\log \psi$, where $\psi$ is the ground state. When $V$ is the scalar curvature, this was studied by Schoen and myself extensively. These problems are naturally related to conformal deformation, stability of minimal surfaces, etc. (The works of D. Fischer-Colbrie and Schoen [163], Micallef [361], Schoen-Yau $[425,431]$ on stable minimal surfaces all depend on understanding of spectrum of this operator.) The parabolic version appears in the recent work of Perelman.

There is important work of Fefferman, Phong, E. Lieb, Duistermaat, Guillemin, Melrose, Colin de Verdier, Taylor, Toth, Zelditch, Sarnak on understanding the spectrum of Laplacian from the point of view of semi classical analysis (see, e.g. $[161,155,225,418])$. Some of their ideas can be found in the geometric optic analysis of J. Keller. The fundamental work of Duistermaat and Hörmander [154] on propagation of singularities was also used extensively. There has been a lot of progress on the very difficult question of determining when one "Can here the shape of a drum" by, among others, Melrose (see [360]), Guillemen [211], Zelditch [559]. (Two dimensional counterexamples were given by GordonWebb-Wolpert [189], Wilson, Szaboó)

Natural generalizations of harmonic or holomorphic functions are harmonic or holomorphic sections of bundles with connections. The most important bundles are the exterior power of cotangent bundles. Using the Levi-Civita connection, harmonic sections are harmonic forms which, by the theory of de Rham and Hodge, give canonical representation of cohomology classes. The major research on harmonic forms comes from Bochner's vanishing theorem [43]. But our understanding is still poor except for one forms. If the manifold is Kähler, differential forms can be decomposed further to $(p, q)$-forms and Kodaira vanishing theorem [268] yields much more powerful information.

In order to demonstrate the idea behind determining the structure of manifolds by function theory, I was motivated to generalize the uniformization theory of Riemann surface to higher dimensions when I was a graduate student. During this period, I was influenced by some of the works of Greene-Wu [192] in formulating these conjectures. Green and $\mathrm{Wu}$ were interested in knowing wether 
the manifolds are Stein or not.

When the complete Kähler manifold is compact with positive bisectional curvature, this is the Frankel conjecture, as was proved independently by Mori [374] and Siu-Yau [458]. Both arguments depend on the construction of rational curves of low degree. When the manifold has nonnegative bisectional curvature and positive Ricci curvature, Mok-Zhong [372] and Mok [368], using the ideas of Bando [25] in his thesis (which was about the application of Hamilton's Ricci flow to low dimensional manifolds of this type) proved that the manifold is Hermitian symmetric.

When a complete Kähler manifold is noncompact with positive bisectional curvature, I conjectured that it must be biholomorphic to $\mathbb{C}^{n}$ (see [546]). Siu-Yau [457] made the first attempt to prove such a conjecture by using the $L^{2}$-method of Hörmander [241] to construct holomorphic functions with slow growth. (Note that Hörmander's method goes back to Kodaira, which was also generalized by Calabi-Vesentini [68].) Singular weight functions were used in this paper and later much more refined arguments were developed by Nadel [378] and Siu [456] using what is called the multiplier sheaf method, which also found important applications in algebraic geometry.

This work of Siu-Yau was followed by Siu-Mok-Yau [369] and Mok [366, 367] under assumptions about the decay of curvature and volume growth of volume. Shi $[439,440,441]$ introduced Hamilton's Ricci flow to study my conjecture, and his work is fundamental. This was followed by beautiful works of Cao [72, 73], Chen-Zhu [89, 90] and Chen-Tang-Zhu [87]. Assuming the manifold has maximal Euclidean volume growth and bounded curvature, Chen-Tang-Zhu [87] (for complex dimension two) and then $\mathrm{Ni}$ [381] (for all higher dimension) were able to prove the manifold can be compactified as a complex variety. Last year, Albert Chau and Tam [80] were finally able to settle the conjecture assuming maximal Euclidean volume growth and bounded curvature. An important lemma of L. Ni [381] was used, where a conjecture of mine (see [549] or the introduction of [381]) was proved. (It says that maximal volume growth implies scalar curvature quadratic decay in the average sense.)

While we see great accomplishment in the case of positive curvature, very little is known on the case when the Kähler manifold is simply connected and complete with strongly negative curvature. It is conjectured to be a bounded domain in $\mathbb{C}^{n}$. (Some people told me that Kodaira considered similar problem. But I cannot find appropriate reference.) The major problem is to construct bounded holomorphic functions.

The difficulty of construction of bounded holomorphic functions is that the basic principle of $L^{2}$-method of Hörmander come from Kodaira's vanishing theorem. It is difficult to go from functions in weighted $L^{2}$ space to bounded functions. 
In this connection, I was able to show that non-trivial bounded holomorphic functions do not exist on a complete manifold with non-negative Ricci curvature [545].

If the manifold is the universal cover of a compact Kähler manifold $M$ which has nontrivial topological map to a compact Riemann surface with genus $>1$, then one can construct bounded holomorphic function, using arguments of Jost-Yau [256]. In particular, if $M$ has a surjectively topological map to product of Riemann surfaces with genus $>1$ and nontrivial topological degree, the universal cover should have a good chance to be a bounded domain.

Of course, this kind of construction is based on the fact that holomorphic functions are harmonic. Certain rigidity based on curvature forced the converse to be true. For functions, the target space has no topology and rigidity is not expected. Bounded Harmonic functions can not be constructed by solving Dirichlet problem unless some boundary data is assumed. This would make good sense if the boundary has nice CR structure. Indeed, for odd dimensional real submanifold in $\mathbb{C}^{n}$ which has maximal complex linear subspace on each tangent plane, Harvey-Lawson [222, 223] proved the remarkable theorem that they bound complex submanifolds. Unfortunately the boundary of a complete simply connected manifold with bounded negative curvature does not have smooth boundary. It will be nice to define a CR structure on such a singular boundary. One may mention the remarkable work of Kuranish [280, 281, 282] on embedding of abstract CR-structure.

A very important bundle is the bundle of spinors. The Dirac operator acting on spinors is a major geometric operator. Atiyah-Singer were the first mathematicians to study it in geometry and by thoroughly understanding the Dirac operator, they were able to prove their celebrated index theorem [15]. The vanishing theorem of Lichnerowicz [318] on harmonic spinors over spin manifolds with positive scalar curvature gives strong information. Through Atiyah-Singer index theorem, it gives vanishing theorem for the $\hat{A}$-genus and the $\alpha$ invariants for spin manifolds with positive scalar curvature. The method was later sharpened by Hitchin [229] for $K 3$ surfaces and Gromov-Lawson [204, 205]. An effective use of this theorem for $\operatorname{spin}_{\mathbb{C}}$ structure for four dimensional manifold is important for Seiberg-Witten theory. Lawson-Yau [285] were able to use Lichnerowicz's work coupled with Hitchin's work to prove a large class of smooth manifolds have no smooth non-abelian group action and, by using modular forms, K. F. Liu proved a loop space analogue of the Lawson-Yau's theorem for the vanishing of the Witten genus in [331].

On the basis of the surgery result of Schoen-Yau [425, 428] and Gromov-Lawson $[204,205]$, one should expect that a suitable converse to Lichnerowicz's theorem exists. The chief result is that surgery on spheres with codimension $\geq 3$ preserves class of metrics with positive scalar curvature. Once geometric surgery 
is possible, standard works on cobordism theory allow one to deduce some existence result for simply connected manifolds with positive scalar curvature. The best work is due to Stolz [466] who gave a complete answer in the case of simply connected manifolds with dimension greater then 4 . I also suggested that one should perform surgery on asymptotic hyperbolic manifold with conformal boundary whose scalar curvature is positive. This is related to the recent work of Witten-Yau [534] on the connectedness of the conformal boundary.

The study of metrics with positive scalar curvature is the first important step in understanding the question of positive mass conjecture in general relativity. Schoen-Yau $[426,430]$ gave the first proof using ideas of minimal surfaces. Three years later, Witten [527] gave a proof using harmonic spinors. Schoen-Yau then generalized their argument to higher dimensional manifolds with positive scalar curvature [428]. Subsequently Gromov-Lawson [204, 205] observed that Lichnerowicz theorem can be coupled with fundamental group and give topological obstructions for metric with positive scalar curvature. This work was related to Novikov conjecture where many authors, including Lusztig [342], Rosenberg [404], Weinberger [524] and G. L. Yu [557] made contributions.

Besides its importance on demonstrating the stability of Minkowski spacetime, positive mass conjecture was used by Schoen [419] in a remarkable manner to finish the proof of the Yamabe problem where Trudinger [507] and Aubin [16] made substantial contributions.

Korevaar-Mazzeo-Pacard-Schoen [276] developed powerful method to understand Nirenberg's problem on prescribed curvature. It was followed by Chen-Lin [93], Chang-Gursky-Yang [79]. However, the integrability condition of Kazdan and Warner is still not fully understood.

The relation between spectrum of manifolds and their global topology is very deep. Atiyah-Bott-Hirzebruch-Singer were the first to study this relationship. Atiyah-Bott-Patodi [9] related the heat kernel expansion to a proof of the local index theorem. Atiyah-Patodi-Singer $[12,13,14]$ initiated the study of spectrum flow and gave important global spectral invariants on odd dimensional manifolds. These global invariants become boundy terms for $L^{2}$-index theorem developed by Atiyah-Donnelly-Singer [10] and Mark Stern [465]. Witten $[528,529]$ has introduced supersymmetry and analytic deformation of de Rham complex to Morse theory, and thereby revealed a new aspect of the connection between global geometry and theoretical physics. Witten's work has been generalized by Demailly [139], Bismut-Zhang[41, 42] to study holomorphic Morse inequality and analytic torsion. Novikov [383] also studied Morse theory for one forms. Witten's work on Morse theory inspired the work of Floer (see, e.g., $[164,165,166]$ ) who used his ideas in Floer cohomology to prove Arnold's conjecture in case the manifold has vanishing higher homotopic group. Floer's theory is related to knot theory (through Chern-Simon's theory [109]) on three 
manifolds. Atiyah, Donaldson, Taubes, Dan Freed, P. Braam, and others (see, e.g., [6, 479, 53, 167]) all contributed to this subject. Fukaya-Ono [177], Oh [384], Kontsevich [272], Hofer-Wysocki-Zehnder [237], G. Liu-Tian [330], all studied such a theory in relation to symplectic manifold. Some part of Arnold's conjecture was claimed to be proved. But a completely satisfactory proof has not been forthcoming.

One should also mention here the very important work of Cheeger [82] and Müller [375] in which they verify the conjecture of Ray-Singer about the relation between analytic torsion with combinational structure of the manifold. The fundamental idea of Ray-Singer [406] on holomorphic torsion are still being vigorously developed in beautiful work of Vafa et al [37]. Later work on analytic torsion was done by Quillen, Todorov, Kontsevich, Borcherds , Bismut, Lott, Zhang and Z. Q. Lu (see [40] and it's reference, [253], [47, 48]). The local version of the index theorem due to Atiyah-Bott-Patodi [9] was later extended in an sophisticated way by Bismut [39] to index theorem for family of elliptic operators. (The local index argument dates back to the foundational work of Mckean-Singer [352].) Quillen's work had been important in this direction (see, e.g. [402, 403]). The study of elliptic genus by Witten [530], Bott-Taubes [50], Taubes [478], K. F. Liu [332] and M. Hopkins [240] has built a bridge between topology and modular form.

It should not be forgotten that, during the seventies, Bott [49] and Atiyah-Bott [8] developed the localization formula for equivariant cohomology. This has been a very important tool. For example, it is used in the most powerful way by K. F. Liu and his coauthors on several topics: the mirror principle (LianLiu-Yau [314, 315, 316, 317]), topological vertex (Li-Liu-Liu-Zhou [295]), etc. Idea of applying localization to enumerative geometry was initiated by Kontsevich [271] and later by Givental [186] and Lian-Liu-Yau [314] independently. These works solve the identities conjectured by Candelas et al [70] based on mirror symmetry, and provide good examples of the ways in which conformal field theory can be a source of inspiration when looking at classical problems in mathematics.

Holomorphic sections of holomorphic line bundles have always been important in algebraic geometry. Riemann-Roch formula coupled with vanishing theorems gave very powerful existence results for sections of line bundle. The Kodaira embedding theorem [269] which said that every Hodge manifold is projective has initiated the theory of holomorphic embeddings of Kähler manifolds. For example, Hirzebruch-Kodaira [228] proved that every odd (complex) dimensional Kähler manifold diffeomorphic to projective space is biholomorphic to projective space. 
Given an orthonormal basis of holomorphic sections of a line bundle, we can embedding it into projective space. The induced metric is the Bergman metric associated to the line bundle. Note that the original definition of Bergman metric used the canonical line bundle and $L^{2}$-holomorphic sections.

In the process of trying to understand the relation between stability of a manifold and existence of Kähler Einstein metric, I [548] proposed that every Hodge metric can be approximated by Bergman metric as long as we allow the power of the line bundle to be large. Following the ideas of the paper of Siu-Yau [457], Tian [497] proved the $C^{2}$ convergence in his thesis under my guidance. My other student W. D. Ruan [405] then proved $C^{\infty}$ convergence in his thesis. This work was followed by $\mathrm{Lu}$ [338], Zelditch [558] and Catlin [77] who observed that the asymptotic expansion of the kernel function follows from some rather standard expression of the Szegö kernel, going back to Fefferman [160] and Boutet de Monvel-Sjöstrand [51] on some circle bundle over the Kähler manifold.

Kodaira's proof of embedding Hodge manifolds by sufficiently high power of positive line bundle is not effective in terms of the power. Matsusaka [348] and later Kollár [270], Siu [454] were able to make the power effective. Demailly $[140,141]$ and Siu $[454,456]$ were able to give remarkable theorems which nearly solve the famous Fujita conjecture [174] (see also Ein and Lazarsfeld [157]). Siu's powerful method also leads to a proof of the deformation invariance of plurigenera of algebraic manifolds [455].

\section{Mappings between manifolds and rigidity of geometric structures}

Another direction to generalize the concept of functions is the study of maps between manifolds. One can define the energy of maps between manifolds and its critical point is called harmonic map. Eells-Sampson [156] was the first one to prove existence of such maps in their homotopy class if the image manifold has non-positive curvature.

Even when I was working on my thesis on manifolds with non-positive curvature in relation to their fundamental group, I realized that it is possible to use harmonic map to reprove some of the theorems in my thesis. I also realized the importance of harmonic maps in the study of rigidity questions in geometry. At that time, the most famous theorem was Mostow's rigidity theorem [376]. In 1976, I proved the Calabi conjecture and applied the Kähler Einstein metric and the Mostow rigidity theorem to prove uniqueness of complex structure on the quotient of the ball [542]. I therefore proposed to use harmonic map to prove the rigidity of complex structure for Kähler manifolds with strongly negative curvature. I proposed this to Siu who carried out the idea when the image manifold satisfies a curvature condition [451] stronger than what I proposed. 
Further result was obtained by Jost-Yau [257], Mok-Siu-Yeung [370] on the proof of superrigidity theorem of Margulis [346], improving an earlier result of Corlette [133] who proved superrigidity for certain rank one locally symmetric space. Complete understanding of superrigidity for the quotient of complex ball is not yet available. One needs to find more structures for the harmonic map which reflect the underlying structure of the manifold. The ideas were based on an argument of Matsushima [350] as was suggest by Calabi. (This was a topic discussed by Calabi in the special year on geometry in the Institute for Advanced Study.)

There are ideas of considering discrete analogue of harmonic maps. When the image manifold is a metric space, there is work by Gromov-Schoen [206], Korevaar-Schoen [277] and Jost [255]. When the domain manifold is a simplicial complex, there are articles by Ballmann-Śwwiạtkowski [23] and M. T. Wang $[517,518]$, in which they introduce generalizations of buildings. They also generalized the works of $\mathrm{H}$. Garland [179] on the vanishing of cohomology group for $p$-adic buildings.

Using the concept of center of gravity, Besson-Courtois-Gallot [38] gives a metric rigidity theorem for rank one locally symmetric space. There are also beautiful rigidity inverse problems for metric geometry due to Gerver-Nadirashvili [184], Pestov-Uhlmann [392] on recovering a Riemannian metric when one knows the distance functions between pair of points on the boundary.

Harmonic map behaves especially well for Riemann surface. Morrey was the first one who solved the Dirichlet problem for energy minimizing harmonic map into any Riemannian manifold.

Another major breakthrough was made by Sacks-Uhlenbeck [413] in 1978 where they constructed minimal spheres in Riemannian manifold representing elements in the second homotopy group using a beautiful extension theorem of harmonic map at an isolated point. By pushing their method further, Siu-Yau [458] studied the bubbling process for the harmonic map and made use of it to prove existence of holomorphic spheres under curvature assumptions. As a consequence, they proved the famous conjecture of Frankel that Kähler manifold with positive bisectional curvature is $\mathbb{C} P^{n}$. (This was also proved by Mori [374].)

Gromov [202] then realized that pseudoholomorphic curve for almost complex structure can be used in a similar way to prove rigidity of symplectic structure on $\mathbb{C} P^{n}$. The bubbling process mentioned above was sharpened further to give compactification of the moduli space of pseudoholomorphic maps by Ye [555] and Parker-Wolfson [388]. Based on these ideas, Kontsevich [271] introduced concept to stable maps and their compactification. 
The formal definitions of Gromov-Witten invariants and quantum cohomology were based on these developments and the ideas of physicists. For example, quantum cohomology was initiated by Vafa (see, e.g., [513]) and his coauthors (the name was suggested by Greene and me). Associativity in quantum cohomology was due to four physicists WDVV [532, 142]. The mathematical treatment (due to Ruan [411] and subsequently by Ruan-Tian [412]) followed the gluing ideas of the physicists. Ruan-Tian made use of ideas of Taubes [477]. But important points were overlooked. A. Zinger [563, 564] has recently completed these arguments.)

In close analogy with Donaldson's theory, one needs to introduce the idea of virtual cycle in the moduli space of stable maps. The algebraic setting of such concept is deeper than the symplectic case and is more relevant to the development for algebraic geometry. The major idea was due to Jun Li who also did the algebraic geometric counterpart of Donaldson's theory (see [292, 296]). (The same comment applies to the so called relative Gromov-Witten invariant, where Jun Li made the vital contribution in the algebraic setting [293, 294].) The symplectic version of Li-Tian [297] ignores difficulties, many of which are completed recently by A. Zinger [563, 564].

Schoen-Yau [429] studied in 1976 the meaning of the action of an $L_{1}^{2}$ map on the fundamental group of a manifold. It was used to prove existence of harmonic map with prescribed action on the fundamental group. Recently F. H. Lin developed this idea further [325]. He studied extensively geometric measure theory on the space of maps (see, e.g., $[322,324]$ ). The action on the second homotopy group is much more difficult to understand. I think there should exist a harmonic map with nontrivial action on the second homotopic group if such continuous map exists.

The energy functional for maps from $S^{2}$ into a manifold does not quite give rise to Morse theory. But the perturbation method of Sacks-Uhlenbeck does provide enough argument for Micallef-Moore [362] to provide some structure theorem for manifolds with positive isotropic curvature.

The major work of harmonic maps on regularity theory in higher dimension is due to Schoen-Uhlenbeck [421, 422]. Leon Simon (see [446]) made deep contribution to the structure of harmonic maps or minimal subvarieties near their singularity. This was followed by F. H. Lin [324].

In early eighties, C. H. Gu [208] initiated a new branch of interest for harmonic maps. He was the first one to study harmonic map when the domain manifold is the Minkowski spacetime. It was the beginning of a new subject called wave maps. This subject was studied extensively by Christodoulou , Klainerman, Tao, Tataru and M. Struwe (see, e.g., [118, 265, 474, 475, 438]). 
Uhlenbeck [511] used techniques from integrable systems to construct harmonic maps from $S^{2}$ to $U(n)$, Bryant [57] and Hitchin [232] also contributed to related constructions using twistor theory and spectral curves. These inspired Burstall, Ferus, Pedit and Pinkall [62] to construct harmonic maps from a torus to any compact symmetric space. In a series of papers, Terng and Uhlenbeck [490, 491] used loop group factorizations to solve the inverse scattering problem and to construct Bäcklund transformations for soliton equations, including Schrödinger maps from $\mathbb{R}^{1,1}$ to a Hermitian symmetric space.

\section{Submanifolds defined by variational principles}

Given a conformal structure on a Riemann surface $\Sigma$, a harmonic map from $\Sigma$ to a fixed Riemannian manifold may minimize energy within a certain homotopy class. However, it may not be conformal and may not be a minimal surface. In order to obtain a minimal surface, we need to vary the conformal structure on $\Sigma$ also. Since the space of conformal structures on a surface is not compact, one needs to make sure the minimum can be achieved.

If the map $f$ induces an injection between the fundamental group of the domains, Schoen-Yau proved the energy is proper on the moduli space by making use of a theorem of Linda Keen. (They developed the theory of topology of $L_{1}^{2}$ map.) Hence they proved the existence of incompressible minimal surfaces [425]. As a product of this argument, it is possible to find a nice exhaustion function for the Teichmüller space. Michael Wolf [535] was able to use harmonic maps to give a compactification of Teichmüller space which he proves to be equivalent to the Thurston compactification. S. Wolpert studied extensively the behavior of the Weil-Petersson metric (see Wolpert's survey [536]). Royden [410] proved that the Teichmüller metric is the same as the Kobayashi metric. C. McMullen [377] introduced a new Kähler metric on the moduli space which can be used to demonstrate that the moduli space is hyperbolic in the sense of Gromov. The great detail of comparison of various intrinsic metrics on the Teichmuller space had been a major problem [548]. It was accomplished recently in the works of Liu-Sun-Yau [333, 334]. Actually Liu-Sun-Yau introduced new metrics with bounded negative curvature and geometry and found the stability of the logrithmatic cotangent bundle of the moduli spaces. Schoen-Yau [424] also proved that degree one harmonic maps are one to one if the image surface has non-positive curvature. Results of this type work only for two dimensional surfaces.

There is a long and rich history on minimal surfaces in Euclidean space. Recent articles include works by Meeks, Osserman, Lawson, Gulliver, White, Hildebrandt, Rosenberg, Collin, Hoffman, Karcher, Ros, Colding, Minicozzi, Rodríguez, Nadirashvili and others (see the reference in Colding and Minicozzi's survey [131]) on embedded minimal surfaces in Euclidean space. They 
come close to classifying complete embedded minimal surfaces and a good understanding of complete minimal surface in a bounded domain. For example, Meeks-Rosenberg [353] proved that the plane and helicoid are the only properly embedded simply connected minimal surfaces in $\mathbb{R}^{3}$.

In a series of papers started in 1978, Meeks-Yau [355, 356, 357, 358] settled a classical conjecture that the Douglas solution for the Plateau problem is embedded if the boundary curve is a subset of a convex boundary. (One should note that Osserman [386] had already settled the old problem of non-existence of branched points for the Douglas solution while Gulliver [213] proved nonexistence of false branched point.) We made use of area minimizing property of minimal surfaces to prove these surfaces are equivariant with respect to the group action. Embedded surfaces which are equivariant play important roles for finite group actions on manifolds. Coupling with a theorem of Thurston, we can then prove the Smith conjecture [554] for cyclic groups acting on the spheres: that the set of fixed points is not a knotted curve.

Meeks-Yau used Douglas-Morrey solution of the Plateau problem by fixing the genus of the surfaces. There is a difficult problem of minimizing area letting the genus to be arbitrary large. This was settled by Hardt-Simon [221]. In the other direction, Almgren-Simon [3] succeeded to minimize embedded disk in Euclidean space. The technique was used by Meeks-Simon-Yau [354] to prove existence of embedded minimal spheres enclosing fake ball. This theorem has been important to prove that the universal covering of an irreducible three manifold is irreducible. They also gave conditions for existence of embedding minimal surfaces of higher genus. This work was followed by topologists Freedman-Hass-Scott [169]. Pitts [395] used mini-max argument for varifolds to prove existence of embedded minimal surfaces. Simon-Smith (unpublished) managed to prove existence of an embedded minimax sphere for any metric on three spheres. Jost [254] was able to extend it to find four minimax spheres. Pitts-Rubinstein (see, e.g., [396]) continued to study such mini-max surfaces. Three manifold topologists were able to adapt the ideas of Meeks-Yau to handle combinational type minimal surfaces and gave applications in three manifold topology.

The most recent works of Colding and Minicozzi [126, 127, 128, 129] on lamination by minimal surfaces and estimates of minimal surfaces without area bound are remarkable and may eventually give good applications in the topology of three manifolds. In fact, they [130] did make contributions to Hamilton's Ricci flow to bound the time evolution before it hits a singularity.

Higher dimensional minimal subvarieties are very important for geometry. There are works due to Federer-Fleming [159], Almgren [2] and Allard [1]. The attempt to prove the Bernstein conjecture, that a minimal graphs are linear, was a strong drive for its development. Bombieri, De Giorgi and Giusti [46] 
found the famous counterexample to the Bernstein problem. It initiated a great deal of interest on area minimizing cone (as graph must be area minimizing). Schoen-Simon-Yau [420] found a completely different approach to the proof of Bernstein problem in low dimensions. This paper on stable minimal hypersurfaces originated many developments on curvature estimates for codimensional one stable hypersurfaces. There are also works by L. Simon with Caffarelli and Hardt [64] on constructing minimal hypersurfaces by deforming stable minimal cones. Recently N. Wickramasekera [525, 526] did some deep work on stable minimal (branched) hypersurfaces which generalizes Schoen-Simon-Yau.

The best regularity result for higher codimension was due to F.Almgren where he proved that for any area minimizing variety, the singular set has codimension at least two. How such a result can be used for geometry remains to be seen. Perhaps the most important question is the Hodge conjecture: whether a multiple of a $(p, p)$ type cohomology class in a projective manifold can be represented by an algebraic cycle. An attempt was made by Lawson, making use of an observation due to J. Simons that any stable subvariety in complex projective space is algebraic [283]. The problem of how to use the hypothesis of $(p, p)$ type has been difficult. In general, the algebraic cycle is not effective. This also creates difficulties for analytic methods. The work of King [263] and Shiffman [443] on complex currents may be relevant.

Perhaps one should generalize the Hodge conjecture to include general $(p, q)$ classes, as it is possible that every cycle in $\bigoplus_{i=-k}^{k} H^{p-i, p+i}$ is rationally homologous to an algebraic sum of minimal varieties such that there is a $p-k$ dimensional complex space in the normal space for almost every point of the variety.

Note that it may be important to assume the metric to be canonical, e.g. Kähler Einstein metric.

A dual question is how to represent homology class by Lagrangian cycles which are minimal submanifolds also. When the manifold is Calabi-Yau, these are special Lagrangian cycles. Since they are supposed to be dual to holomorphic cycles, there should be an analogue of the Hodge conjecture. For example if $\operatorname{dim}_{\mathbb{C}} M=n$ is odd, any element in $\bigoplus_{i+j=n} H^{i, j}$ should be representable by special Lagrangian cycles up to a rational multiple provided the cup product of it with the Kähler class is zero.

A very much related question is: if the Chern classes of a complex vector bundle are of $(p, p)$ type, does the vector bundle, after adding a holomorphic vector bundle, admit a holomorphic structure? If the above generalization of the Hodge conjecture holds, there should be a similar generalization for the vector bundle. 
These questions had a lot more success for four dimensional symplectic manifolds by the work of Taubes both on the existence of pseudoholomorphic curves [486] and on the existence of anti-self-dual connections [476, 477]. On a Kähler surface, anti-self-dual connections are Hermitian connections for a holomorphic vector bundle. In particular, Taubes gave a method to construct holomorphic vector bundles over Kähler surfaces.

Another important class of minimal varieties is the class of special Lagrangian cycles in Calabi-Yau manifolds. Such cycles were first developed by HarveyLawson [224] in connection to calibrated geometry in Euclidean space. Major works were done by Schoen-Wolfson [423], Yng-Ing Lee [288] and Butscher [63]. One expects Lagrangian cycles to be mirror to holomorphic bundles and special Lagrangian cycles to be mirror to Hermitian-Yang-Mills connections. Hence by the Donaldson-Uhlenbeck-Yau theorem, it is related to stability. The concept of stability for Lagrangian cycles was discussed by Joyce and Thomas. Thomas-Yau [492] had therefore made suggestions about an analogy for the the mean curvature flow for the existence of special Lagrangian cycles based on stability of Lagrangian cycles. Their existence and the relation to mean curvature flow is very interesting. See M. T. Wang [519, 520], Smoczyk [461] and Smoczyk-Wang [462]. The geometry of mirror symmetry was explained by Strominger-Yau-Zaslow in [468] using a family of special Lagrangian tori. There are other manifolds with special holonomy group. They have similar calibrated submanifolds. Conan Leung has contributed to studies of such manifolds and their mirrors (see, e.g. [290, 291]).

Besides minimal surfaces, another important class of surfaces are surfaces with constant mean curvature and also surfaces that minimize the $L^{2}$ norm of the mean curvature. It is important to know the existence of such surfaces in a three dimensional manifold with nonnegative scalar curvature.

The Existence of minimal spheres is related to the existence of black holes. The most effective method was developed by Schoen-Yau [432] using the existence theorem they [427] proved for the equation of Jang. It should be interesting to develop methods to prove existence of stable minimal spheres. Extremum of the Hawking mass is related to minimization of $L^{2}$ norm of mean curvature. Their existence and behavior have not been understood.

For surfaces with constant mean curvature, we have the concept of stability. (Fixing the volume it encloses, the second variation of area is non-negative.) Making use of my work on eigenvalues with Peter Li, Christodoulou and I [119] proved that the Hawking mass of these surfaces is positive. This fact was used by Huisken and I [248] to prove uniqueness and existence of foliation by constant mean curvature spheres for a three dimensional asymptotically flat manifold with positive mass. (We initiated this research in 1986. Ye studied our work and proved existence of similar foliations under various conditions, see 
[556].) After ten years, Tian and Qing [401] reproduced our uniqueness result, but the argument is basically the same.

This foliation was used by Huisken and Yau [248] to give a canonical coordinate system at infinity. It defines the concept of center of gravity where important properties for general relativity are found. The most notable is that total linear momentum is equal to the total mass multiple with the velocity of the center of mass. One expects to find good asymptotic properties of the tensors in general relativity along these canonical surfaces. We hope to find a good definition of angular momentum based on this concept of center of gravity so that global inequality like total mass can dominate the square norm of angular momentum. Some preliminary attempt was made by my former student X. Zhang [561], but his definition does not have good physical motivation and does not seem to lead to anything significant at this time.

In the paper of Huisken-Yau [248], the uniqueness of foliation was improved to the uniqueness of a single closed hypersurface with constant mean curvature enclosing a compact set of the three manifold. This compact set was defined in terms of the mean curvature of the hypersurface in [248]. As a side remark, Huisken-Yau proposed to enlarge this neighborhood of uniqueness to be independent of the choice of the mean curvature. While this improvement for a single hypersurface has some interest, it has little to do with the above applications in general relativity. In particular, it is not needed to define the important concept of center of gravity. This proposal of Huisken-Yau was observed to be true by Qing-Tian [401] based on the same argument of Huisken-Yau and a simple integral formula.

The idea of using foliation of surfaces satisfying various properties (constant Gauss curvature, for example) to study three manifolds in general relativity is also developed by R. Bartnik [30]. His idea of quasi-spherical foliation gives a good parametrization of a large class of metrics with positive scalar curvature.

Some of these ideas were used by Shi-Tam [442] to study quantities associated to spheres which bound three manifolds with positive scalar curvature. Such a quantity is realized to be the quasi-local mass of Brown-York [56]. At the same time, M. Liu and Yau [328, 329] were able to define a new quasi-local mass for general spacetimes in general relativity, where some of the ideas of Shi-Tam were used. Further works by M. T. Wang and myself generalize Liu-Yau's work by studying surfaces in hyperbolic space-form.

My interest in quasi-local mass dates back to the paper that I wrote with Schoen [432] on the existence of a black hole due to condensation of matter. It is desirable to find a quasi-local mass which includes effect of matter and nonlinear effect of gravity. Hopefully one can prove that when such a mass is larger than a constant multiple of square root of the area, a black hole forms. This has not 
been achieved yet.

The argument of Huisken-Yau depends on the mean curvature flow. There is another flow called the inverse mean curvature which was proposed by Geroch [183] to understand the Penrose conjecture relating the mass with the area of the black hole. Such procedure was finally carried out by Huisken-Ilmanen [245] when the scalar curvature is non-negative. There was a different proof by H. Bray [55] subsequently. Mean curvature flow for varifolds was initiated by Brakke [54]. The level set approach was studied by many people: S. Osher, L. Evans, Giga, etc (see [385, 158, 96]). Huisken [243, 244] did the first important work when the initial surface is convex. His recent work with Sinestrari $[246,247]$ on mean convex surfaces is remarkable and gives a good understanding of the structure of singularities of mean curvature flow.

Natural elliptic problems with higher order are difficult to handle. Affine minimal surfaces and Willmore surfaces are such examples. L. Simon [445] did make important contribution to the regularity of the Willmore surfaces.

Most elliptic problems have a parabolic version. But it is not a trivial matter to find good quantities to apply maximal principle on a manifold. Besides the work of Li-Yau [309], I expect the Cheng-Yau gradient estimates for maximal slice and affine spheres to have a parabolic version. On the other hand, not much progress is known on the Calabi flow (see Chang's survey [78]) for Kähler metrics. They are higher order problem where maximal principle has not been effective. An important contribution was made by Chrúsciel [120] for Riemann surface.

The dynamics of Einstein equations for general relativity is a very difficult subject. The Cauchy problem was considered by many people: Lichnerowicz, Choquet-Bruhat, York, Moncrief, Friedrich, Christodoulou, Klainerman, H. Lindblad, Dafermos (see, e.g., [319], [115], [114], [172], [116], [264], [326], [136]). But the global behavior is still far from being understood. The major unsolved problem is to formulate and prove the fundamental question of Penrose on Cosmic censorship. I suggested to Klainerman to consider small initial data for the Einstein system. The treatment of stability of Minkowski spacetime was accomplished by Christodoulou-Klainerman [117] under small fall off conditions. Recently Lindblad and Rodnianski [326] gave a simpler proof. A few years ago, N. Zipser (Harvard thesis) added Maxwell equation to gravity and still proved stability of Minkowski spacetime. Stability for Schwarzschild or Kerr solutions is far from being known. Finster-Kamran-Smoller-Yau [162] had studied decay properties of Dirac particles with such background. The works are rather nontrivial and does indicate stability of these classical spacetime. 
There is extensive literature on spacelike hypersurfaces with constant mean curvature. The foliation defined by them can give interesting dynamical information of spacetime. These surfaces are interesting even for $\mathbb{R}^{3,1}$. Treiberges studied it extensively [506]. Li, Choi-Treibergs [111] and T. Wan [515] observed that the Gauss maps of such surfaces give very nice examples of harmonic maps mapping into the disk. Recently Fisher and Moncrief used them to study the evolution equation of Einstein in $2+1$ dimension.

\section{Construction of geometric structures on bundles and manifolds}

It is a fundamental question to build geometric structures over a given manifold. In general, the group of topological equivalence that leaves invariant this geometric structure should be a special group. With the exception of symplectic structures, these groups are usually finite dimensional. When the geometric structure is unique (up to equivalence), it can be used to produce key informations about the topological structure.

The study of special geometric structures dates back to Sophus Lie, Klein and Cartan. In most cases, we like to be able to parallel transform vectors along paths so that we can define the concept of holonomy group.

When the holonomy group is not compact, there are examples of projective flat structure, affine flat structure and conformally flat structure. It is not a trivial matter to determine which topological manifolds admit such structures. Since the structure is flat, there is a unique continuation property and hence one can construct a developing map from a suitable cover of the manifold to the real projective space, the affine space and the sphere respectively. The map gives rise to a representation of the fundamental group of the manifold to the real projective group, the special linear group and the Möbius group respectively. This holonomic representation gives a great deal of information for the geometric structure. Unfortunately, the map is not injective in general. In case it is injective, the manifold can be obtained as quotient of a domain by a discrete subgroup of the corresponding Lie group. In this case, a lot more can be said about the manifold as the theories of partial differential equations and discrete group can play important roles. For example, if a projective flat manifold can be projectively embedded as a bounded domain, Cheng-Yau [104] were able to construct a canonical metric from the real Monge-Ampère equation which generalizes the Hilbert metric. When the manifold is two dimensional, there are works of Wang [516] and Loftin [335] on how to associate such metrics to a conformal structure and a holomorphic section of the cubic power of canonical bundle. This is a beautiful theory related to the hyperbolic affine sphere mentioned in the first section. 
There are fundamental works of Choi, Goldman (see the reference of Choi and Goldman's paper [113]) and Hitchin [233] and others on the geometric decomposition and moduli of projective structures on Riemann surfaces. It should be interesting to extend them to three or four dimensional manifolds.

It is a difficult question to determine which manifolds admit affine structures. It is an open question whether the Euler number of such spaces is zero although great progress was made by Sullivan [470]. Goldman [187] has also found topological constrains on three manifolds in terms of fundamental group. The difficulty arises as there is no useful metric that is compatible with the underlining affine structure. This motivated Cheng-Yau [105] to define the concept of affine Kähler metric.

When Cheng and I considered the concept of affine Kähler metric, we thought that it was a natural analogue of Kähler metrics. However, compact nonsingular examples are not bountiful. Strominger-Yau-Zaslow [468] proposed the construction of mirror manifolds by constructing the quotient space of a CalabiYau manifold by a special Lagrangian torus. At the limit of large Kähler class, it was pointed out by Hitchin [234] that the quotient space admits a natural affine structure with a compatible affine Kähler structure. But in general, we do expect singularities of such structure. It now becomes a deep question to understand what kind of singularity is allowed and how we build the CalabiYau manifold from such structures. Loftin-Yau-Zaslow [336] have initiated the study of the structure of "Y" singularity. Hopefully one can find existence theorem for affine structures over compact manifolds with prescribed singularities along codimensional two stratified submanifolds.

Construction of conformally flat manifolds is also a very interesting topic. Similar to projective flat or affine flat manifolds, there are simple constraints from curvature representation for the Pontrjagin classes. The deeper problem is related to the understanding of the fundamental group and the developing map. When the structure admits a conformal metric with positive scalar curvature, Schoen-Yau [433] proved the rather remarkable theorem that the developing map is injective. Hence such manifold must be the quotient of a domain in $S^{n}$ by a discrete subgroup of Möbius transformations. It would be interesting to classify such manifolds. In this regard, the Yamabe problem as was solved by Schoen [419] did provide a conformal metric with constant scalar curvature. One hopes to be able to use such metrics to control the conformal structure. Unfortunately the metric is not unique and a deep understanding of the moduli space of conformal metrics with constant scalar curvature should be important.

It is curious that while bundle theory was used extensivly in Riemannian geometry, it has not been used in these geometries. One can construct real projective space bundles, affine bundles or sphere bundles by mapping coordinate charts 
to the corresponding model spaces (possible with different dimensions) and gluing the target model spaces together to form natural bundles. One may want to study the associated Chern-Simons forms [109].

An important goal of geometry is to build a canonical metric associated to a given topology. Besides the uniformization theorem in two dimension, the only (spectacular) work in this regard is the geometrization conjecture of Thurston (see [495]).

Thurston made use of ideas from Riemann surface theory, Haken's work [214] on three manifold, Mostow's rigidity [376] and discrete group theory of Lie group to build his program. He made the assumption that an incompressible surface exists in the three manifold. When Hamilton [215] had his initial success on his Ricci flow, I immediately suggested (around 1981) to him to use his flow to break up the manifold. His generalization of the theory of Li-Yau [309] to Ricci flow [216, 217] and his seminal paper in 1996 [218] on breaking up manifold mark cornerstone of the program. Perelman's recent idea [389, 390] built on these two works and has gone deeply to the problem. Detailed discussions are being pursued by Zhu, Cao, Hamilton and Huisken in the past two years. Hopefully it may lead to the final settlement of Thurston's program. This theory of Hamilton and Perelman should be considered as a crowning achievement of geometric analysis in the past thirty years. Most ideas developed in this period by geometric analysts are used.

Hyperbolic metrics have been used by topologists to give invariants for three dimensional manifolds. Thurston [494] observed that the volume of the hyperbolic metric is an important topological invariant. The associated ChernSimons [109] invariant, which is defined mod integers, can be looked upon as a phase for such manifolds. These invariants appeared later in Witten's theory of $2+1$ dimensional gravity [530] and S. Gukov [212] was able to relate them to fundamental questions in knot theory.

The major accomplishment of Thurston, Hamilton, Perelman et al is the ability to create a canonical structure on three manifolds. Such structure has not even been conjectured for four manifolds despite the great success of Donaldson invariants and Seiberg-Witten invariants. Taubes [480] did prove a remarkable existence theorem for self-dual metrics on a rather general class of four dimensional manifolds. Unfortunately their moduli space is not understood and their topological implication is not clear at this moment. Prior to the construction of Taubes, Donaldson-Friedman [151] and LeBrun [286] have used ideas from twistor theory to construct self-dual metrics on connected sum of $\mathbb{C} P^{2}$.

While the paper of Atiyah-Hitchin-Singer [11] laid the algebraic and geometric foundation for self-dual connections, the analytic foundation was laid by Uhlenbeck $[509,510]$ where she established the removable singularity theorem and 
compactness theorem for Yang-Mills connections. This eventually led to the fundamental works of Taubes [477] and Donaldson [143] which revolutionized four manifold topology.

Donaldson's theory started out from anti-self-dual connections on four manifolds. The analogues of it on Kähler manifolds are Hermitian Yang-Mills connections, which was shown by Donaldson [144] for Kähler surfaces and UhlenbeckYau [512] for general Kähler manifolds to be equivalent to polystability of bundles. It was generalized by C. Simpson [447], using ideas of Hitchin [231], to bundles with Higgs fields. It has deep applications to the theory of variation of Hodge structure [448, 449]. Wentworth and Daskalopoulos (see [138]) studied such theory for moduli space of vector bundles over curves. Li-Yau [298] generalized existence of Hermitian Yang-Mills connections to non-Kähler manifolds. (Buchdahl [60] subsequently did the same in the case of complex surfaces.) Li-Yau-Zheng [300] then used the result to give a complete proof of Bogomolov's theorem for class $\mathrm{VII}_{0}$ surfaces. I expect more applications of Donaldson-Uhlenbeck-Yau theory to algebraic geometry.

D. Gieseker [185] developed geometric invariant theory for the moduli space of bundles and introduced Gieseker stability of bundles. Conan Leung [289] introduced the analytic counterpart of such bundles in his thesis under my guidance. While it is a natural concept, there is still an analytic problem to be resolved. (He assumed the curvature of the bundles to be uniformly bounded.)

There were attempts by de Bartolomeis-Tian [32] to generalize Yang-Mills theory to symplectic manifolds and also by Tian [500] to manifolds with special holonomy group. The arguments are not complete and still need to be finished.

For a given natural structure on a manifold, we can often fix a structure and linearize the equation to obtain a natural connection on the tangent bundle. Usually we obtain Yang-Mills connections with extra structure given by the holonomy group of the original structure. It is curious whether an iterated procedure can be constructed to find interesting metric or not. In any case, we can draw analogous properties between bundle theory and metric theory. The concept of stability for bundles is reasonably well understood for the holomorphic category. I believed that for each natural geometric structure, there should be a concept of stability. Donaldson [145] was able to explain stability in terms of moment map, generalizing the work of Atiyah-Bott [7] for bundles over Riemann surfaces. It will be nice to find moment maps for other geometric structures.

The most interesting geometric structure is the Kähler structure. There are two interesting pre-Kähler structures. One is the complex structure and the other is the symplectic structure. The complex structure is a rather rigid structure in two dimension. However it may be much more flexible in dimension 
greater than two. For example, Clemens [122] and Friedman [171] proposed a way to perform complex surgery by blowing down complex submanifolds with codimension greater than one and negative normal bundle. By smoothing out the singularity, one can obtain interesting non-Kähler complex manifold. (LuTian [339] followed these ideas.) These manifolds can play important roles for connecting Calabi-Yau manifolds together as was speculated by M. Reid [407]. In order to give deeper analysis, it will be important to equip them with canonical metrics. (A possible candidate of such metric is the Strominger's system studied by Fu-Yau [173].)

As was pointed out by Smith-Thomas-Yau [460], mirrors of non-Kähler complex manifolds can be symplectic manifolds. They construct a large class of symplectic manifolds with trivial first Chern class by reversing the procedure of Clemens-Friedman. In dimension four, the Betti numbers of such manifolds are determined by $\mathrm{T}$. J. Li [311]. In the last ten years, there has been extensive work on symplectic manifolds, initiated by Gromov [202], Taubes [482, 483, 484, 485]), Donaldson [146, 147, 148] and Gompf [188]. These works are based on the understanding of pseudo-holomorphic curves and Lefschetz fibrations. The most fundamental work is centered around four dimensional manifolds. The major tools are Seiberg-Witten theory $[435,436,533]$ and analysis. The work of Taubes on the existence of pseudo-holomorphic curves and the topological meaning of its counting is one of the deepest works in geometry. Based on this work, Taubes [482] was able to prove the old conjecture that there is only one symplectic structure on the standard $\mathbb{C} P^{2}$. The following question of mine is still unanswered: If $M$ is a symplectic 4-manifold homotopic to $\mathbb{C} P^{2}$, is $M$ symplectomorphic to the standard $\mathbb{C} P^{2}$ ? (The corresponding question for complex geometry was solved by me in [542].) On the other hand, based on the work of Taubes [481], T. J. Li and A. K. Liu [312] did find wall crossing formula for four dimensional manifolds that admit metrics with positive scalar curvature. Subsequently A. Liu gave the classification of such manifolds. (The surgery result by Stolz [466] based on Schoen-Yau-Gromov-Lawson for manifolds with positive scalar curvature is not effective for the four dimensional case.) As another application of the general wall crossing formula in [312], it was proved by T. J. Li and A. Liu in [313] that there is a unique symplectic structure on $S^{2}$-bundles over any Riemann surface. A main result of D. McDuff in [351] is used here.

The Strominger's system [467] does admit supersymmetries. It will be nice to see if an analogous structure can be built on symplectic manifolds with zero first Chern class. Fukaya and Oh [176] have developed an elaborate theory for symplectic manifolds with Lagrangian cycles. Pseudo-holomorphic disks appeared as trace of motions of curves according to Floer theory. Paul Sideal-Thomas [437] and Ruan discussed Fukaya's category in relation to Kontsevich's holomorphic conjecture [273]. One wonders whether Fukaya's theory can help to 
construct canonical metrics for symplectic structures.

The construction of Calabi-Yau manifolds was based on the existence of a complex structure which can support a Kähler structure. A fundamental question is whether an almost complex manifold admits an integrable complex structure when complex dimension is greater than two.

Once we have an integrable complex structure, we can start to search for Hermitian metrics with special properties. As was mentioned earlier, if we would like to have supersymmetries and if we use connections without torsion, then a Kähler metric is the only choice. Further supersymmetry would require it to be Calabi-Yau. However if we do not require the connection to be torsion free, Strominger [467] did derive a set of equations that exhibit supersymmetries without requiring the manifold to be Kähler. It is a coupled system of Hermitian Yang-Mills connections with Hermitian metrics. Twenty years ago, I tried to develop such a coupled system. The attempt was unsuccessful as I restricted myself to Kähler geometry. My student Bartnik with Mckinnon [31] did have a good success in the Lorentzian case. They found non-singular solutions for such a coupled system. (The mathematical rigorous proof was provided by Smoller-Wasserman-Yau-Mcleod [464] and [463]).

The Strominger's system was shown to be solvable in a neighborhood of a Calabi-Yau structure by Jun Li and myself [299]. Fu and I [173] are also able to solve it on complex manifolds which admit no Kähler structure. These manifolds are balanced manifolds and were studied by M. Michelsohn [364]. These manifolds can be used to explain some questions of flux in string theory (see, e.g., $[34,76])$.

The Calabi-Yau structure was used by me and others to solve important problems in algebraic geometry before it appeared in string theory. For example, the proof of Torelli theorem for K3 surface due to Todorov[504]-Siu[453] and the surjectivity of the period map of K3 surface due to Siu[452]-Todorov[504] are important works for algebraic surfaces. The proof of the Bogomolov[44]Tian[496]-Todorov[505] theorem also requires the metric.

In my talk [544] in the Congress in 1978, I outlined the program of classifying noncompact Calabi-Yau manifolds. Some of these works were written up by Tian-Yau [502, 503]. During the period of 1984, there was a strong request by string theorist to construct Calabi-Yau threefolds with Euler number equal to \pm 6 . I [547] constructed such manifold with $\mathbb{Z}_{3}$ fundamental group by taking the quotient of a bi-degree $(1,1)$ hypersurface in the product of two cubics. Soon afterwards, more examples were constructed by Tian and myself [501].

The existence of a Kähler Einstein metric with negative scalar curvature was proved by Aubin [18] and me [543] independently. I [542] did find important 
applications of it to solve classical problems in algebraic geometry, e.g., the uniqueness of complex structure over $\mathbb{C} P^{2}$ [542], the Chern number inequality of Miyaoka[365]-Yau[542] and the rigidity of algebraic manifolds biholomorphic to Shimura varieties. The problem of the existence of Kähler Einstein metrics with positive scalar curvature in the general case is not solved. However, my proof of the Calabi conjecture already provided all the necessary estimates except some integral estimate on the unknown. This of course can be turned into hypothesis. I conjectured that an integral estimate of this sort is related to stability of manifolds. Tian [499] called it K-stability. Mabuchi's functional [345] made the integral estimate to be more intrinsic and it gives rise to a natural variational formulation of the problem. Siu has pointed out that the work of Tian [498] on two dimensional surfaces is not complete. The work of Nadel [378] on multiplier ideal sheaf did give interesting methods for the subject.

For Kähler Einstein manifolds with positive scalar curvature, it is possible that they admit continuous group of automorphisms. Matsushima [349] was the first one to observe that such a group must be reductive. Futaki [178] introduced a remarkable invariant and proved that it must vanish for such manifolds. In my seminars in the eighties, I proposed that Futaki's theorem should be generalized to understand the projective group acting on the embedding of the manifold by high power of anti-canonical embedding and that Futaki's invariant should be relevant to my conjecture [550] relating Kähler Einstein manifold to stability. Tian asked what happens when manifolds have no group actions. I explained that the shadow of the group action is there once it is inside the projective space and one should deform the manifold to a possibly singular one under the projective group to obtain more information. The connection of Futaki invariant with stability of manifolds has finally appeared in the recent work of Donaldson $[149,150]$. One should also mention the recent interesting work of Ross-Thomas [408, 409] on stability of manifolds. There are many contributions on the study of stability of manifold by analytic means. For example, Phong-Strum [393] constructed solutions of certain degenerate Monge-Ampére equations to produce geodesics in the space of Kähler potentials. They [394] also studied convergence of the Kähler-Ricci flow.

My program to understand stability of a manifold through canonical metrics was followed by several authors and the best achievement was made by Donaldson [149] A Kähler metric with constant scalar curvature is equivalent to the fact that the first Chern form is harmonic. The uniqueness theorem for harmonic Kähler metric was due to X. Chen [95], Donaldson [149] and Mabuchi for various cases. (Note that the most important case of the uniqueness of Kähler Einstein metric with positive scalar curvature was due to the remarkable argument of Bando-Mabuchi [26].) The general conjecture for the existence of such harmonic Kähler manifolds based on stability of manifolds is still largely unknown. In my seminar in the mid-eighties, I also dicussed the relation of harmonic Kähler manifolds with stability of manifolds: Several students of mine 
including Tian [497], Luo [341] and Wang [523] had written thesis related to this topic. Prior to them, my former students Bando [25] and Cao [71] had already made attempts to study this problem by Ricci flow. The study of harmonic Kähler metrics with constant scalar curvature on toric variety was initiated by S. Donaldson [150], who proposed to study the existence problem via real Monge-Ampère equation. This was solved by Wang-Zhu [522]. LeBrun and his coauthors [262] also have found special constructions, based on twistor theory, for harmonic surfaces. Bando was in fact interested to study Kähler manifolds with harmonic $i-t h$ Chern form. (it should be interesting to find an analogue of stability for harmonic $i-t h$ Chern class.) Cao taught a course on Kähler metrics in Columbia when S. W. Zhang was working on Arakelov geometry. After he learned about my conjecture relating stability of manifolds to harmonic Kähler metrics, he showed his interest to relate the metric to the height of manifolds. This led him to discuss with our group of students on stability of manifolds in relation to induced metrics from embedding of manifolds into complex projective space [560]. (The last part is related to the above mentioned Bergman metric.) His works on Chow stability in [560] gave strong inputs to my students, including Tian [32]. While Zhang's paper should be credited properly, his work did have nontrivial influence on the later works of Donaldson $[149,150]$.

Besides Käkler manifolds, there are manifolds with special holonomy groups. Holonomy groups of Riemannian manifolds were classified by Berger [35]. The most important ones are $O(n), U(n), S U(n), G_{2}$ and $\operatorname{Spin}(7)$. The first two groups correspond to Riemannian and Kähler geometry respectively. $S U(n)$ corresponds to Calabi-Yau manifolds. A $G_{2}$ manifold is seven dimensional and a $\operatorname{Spin}(7)$ is eight dimensional (assuming they are irreducible manifols). These last three classes of manifolds have zero Ricci curvature. It may be noted that before I [543] proved the Calabi conjecture in 1976, there was no known nontrivial compact Ricci flat manifold. Manifolds with special holonomy group admit nontrivial parallel spinors and they correspond to supersymmetries in the language of physics. The input of ideas from string theory did help to understand these manifolds. However, the very basic question of constructing these structures on a given topological space is still not well understood. In the case of $G_{2}$ and $\operatorname{Spin}(7)$, it was initiated by Bryant (see [58, 59]). The first set of compact examples was given by Joyce [258, 259, 260]. Recently Dai-Wang-Wei [137] proved stability of manifolds with parallel spinors.

The nice construction of Joyce was based on singular perturbation which is similar to the construction of Taubes [476] on anti-self-dual connections. However, it is not global enough to give a good parametrization of $G_{2}$ or $\operatorname{Spin}(7)$ structures. A great deal more works are needed. The beautiful theory of Hitchin $[235,236]$ on three forms and four forms may lead to a resolution of these important problems. 
One can also obtain new geometric structures by imposing some singular structures on a manifold with special holonomy group. For example, if we require a metric cone to admit $G_{2}, \operatorname{Spin}(7)$ or Calabi-Yau structure, the link of the cone will be a compact manifold with special structures. They give interesting Einstein metrics. When the cone is Calabi-Yau, the structure on the odd dimensional manifold is called Sasakian Einstein metric.

There is a natural Killing field called Reeb vector field defined on a Sasakian Einstein manifold. If it generates a circle action, the orbit space gives rise to a Kähler Einstein manifold with positive scalar curvature. However, It need not generate a circle action and J. Sparks, Gauntlelt, Martelli, Waldram [180] gave many interesting explicit examples of non-regular Sasakian Einstein structures. They have interesting properties related to conformal field theory. For quasiregular examples, there were works due to Boyer, Galicki and Kollár [52]. The procedure gave many interesting examples of Einstein metrics on odd dimensional manifolds.

Existence of Einstein metrics on a fixed topological manifold is clearly one of the most important questions in geometry. Any metrics with compact special holonomy group are Einstein. Besides Kähler geometry, we do not know much of their moduli space. For Einstein metric with no special structures, we know only some topological constraints on four dimensional manifolds. There are works due to Berger [36], Gray [191] and Hitchin [230] in terms of inequalities linking Euler number and signature of the manifold. (This is of course based on Chern's work [108] on representation of characteristic classes by curvature forms.) Gromov [201] made use of his concept of Gromov volume to give further constraint. LeBrun [287] then introduced the ideas of Seiberg-Witten invariants to enlarge such classes and gave beautiful rigidity theorems on Einstein four manifolds. Unfortunately it is very difficult to understand moduli space of Einstein metrics when they admit no special structures. For example, it is still an open question of whether there is only one Einstein metric on the four dimensional sphere. M. Wang and Ziller [521] did use symmetric reductions to give many examples of Einstein metrics for higher dimensional manifolds. This is really a very interesting field for geometric analysis.

\section{Basic Philosophy:}

Functions plus subvarieties governed by natural differential equations provide deep insight into geometric structures. The information will provide a way to construct such a geometric structure. It also provides important information for physics, algebraic geometry and topology. Conversely it is vital to learn ideas from these fields. 
Behind such basic philosophy, there are basic invariants to understand how space is twisted. This is provided by Chern Classes [108], which appear in every branch of mathematics and theoretical physics. So far we barely understand the analytic meaning of the first Chern Class. It will take much longer time for geometers to understand the analytic meaning of the higher Chern forms. The analytic expression of Chern Classes for forms have opened up a new horizon for global geometry. Professor Chern's influence on mathematics is forever.

\section{REFERENCES}

[1] W. K. Allard, On the first variation of a varifold: boundary behavior. Ann. of Math. (2) 101(1975), 418-446.

[2] F. J. Almgren Jr., Almgren's big regularity paper. Q-valued functions minimizing Dirichlet's integral and the regularity of area-minimizing rectifiable currents up to codimension 2. With a preface by Jean E. Taylor and Vladimir Scheffer. World Scientific Monograph Series in Mathematics, 1. World Scientific Publishing Co., Inc., River Edge, NJ, 2000.

[3] F. Almgren and L. Simon, Existence of embedded solutions of Plateau's problem. Ann. Scuola Norm. Sup. Pisa Cl. Sci. (4) 6 (1979), no. 3, 447-495.

[4] M. T. Anderson, The Dirichlet problem at infinity for manifolds of negative curvature. J. Differential Geom. 18(1983), no. 4, 701-721 (1984).

[5] M. T. Anderson and R. Schoen, Positive harmonic functions on complete manifolds of negative curvature. Ann. of Math. (2) 121(1985), no. 3, 429-461.

[6] M. Atiyah, New invariants of 3-and 4-dimensional manifolds. The mathematical heritage of Hermann Weyl, 285-299, Proc. Sympos. Pure Math., 48, Amer. Math. Soc., Providence, RI, 1988.

[7] M. F. Atiyah and R. Bott, The Yang-Mills equations over Riemann surfaces. Philos. Trans. Roy. Soc. London Ser. A 308(1983), no. 1505, 523-615.

[8] M. F. Atiyah and R. Bott, The moment map and equivariant cohomology. Topology 23(1984), no. 1, 1-28.

[9] M. Atiyah, R. Bott and V. K. Patodi, On the heat equation and the index theorem. Invent. Math. 19(1973), 279-330.

[10] M. F. Atiyah, H. Donnelly and I. M. Singer, Eta invariants, signature defects of cusps, and values of L-functions. Ann. of Math. (2) 118(1983), no. 1, 131-177.

[11] M. F. Atiyah, N. J. Hitchin and I. M. Singer, Self-duality in four-dimensional Riemannian geometry. Proc. Roy. Soc. London Ser. A 362(1978), no. 1711, 425-461.

[12] M. F. Atiyah, V. K. Patodi and I. M. Singer, Spectral asymmetry and Riemannian geometry. I. Math. Proc. Cambridge Philos. Soc. 77(1975), 43-69.

[13] M. F. Atiyah, V. K. Patodi and I. M. Singer, Spectral asymmetry and Riemannian geometry. II. Math. Proc. Cambridge Philos. Soc. 78(1975), no. 3, 405-432.

[14] M. F. Atiyah, V. K. Patodi and I. M. Singer, Spectral asymmetry and Riemannian geometry. III. Math. Proc. Cambridge Philos. Soc. 79(1976), no. 1, 71-99.

[15] M. F. Atiyah and I. M. Singer, The index of elliptic operators on compact manifolds. Bull. Amer. Math. Soc. 69(1963), 422-433.

[16] T. Aubin, Équations différentielles non linéaires et problème de Yamabe concernant la courbure scalaire. J. Math. Pures Appl. (9) 55 (1976), no. 3, 269-296.

[17] T. Aubin, Problèmes isopérimétriques et espaces de Sobolev. J. Differential Geometry 11(1976), no. 4, 573-598.

[18] T. Aubin, Équations du type Monge-Ampère sur les variétés kähleriennes compactes. C. R. Acad. Sci. Paris Sér. A-B 283(1976), no. 3, Aiii, A119-A121.

[19] T. Aubin, Espaces de Sobolev sur les variétés riemanniennes. Bull. Sci. Math. (2) 100 (1976), no. 2, 149-173. 
[20] W.L. Baily Jr. and A. Borel, A. Compactification of arithmetic quotients of bounded symmetric domains. Ann. of Math. (2) 84(1966) 442-528.

[21] W. Ballmann, On the Dirichlet problem at infinity for manifolds of nonpositive curvature. Forum Math. 1(1989), no. 2, 201-213.

[22] W. Ballmann and F. Ledrappier, The Poisson boundary for rank one manifolds and their cocompact lattices. Forum Math. 6(1994), no. 3, 301-313.

[23] W. Ballmann and J. Świątkowski, On $L^{2}$-cohomology and property (T) for automorphism groups of polyhedral cell complexes. Geom. Funct. Anal. 7(1997), no. 4, 615-645.

[24] W. Ballmann, G. Thorbergsson and W. Ziller, Closed geodesics on positively curved manifolds. Ann. of Math. (2) 116(1982), no. 2, 213-247.

[25] S. Bando, On the classification of three-dimensional compact Kaehler manifolds of nonnegative bisectional curvature. J. Differential Geom. 19(1984), no. 2, 283-297.

[26] S. Bando and T. Mabuchi, Uniqueness of Einstein Kähler metrics modulo connected group actions. Algebraic geometry, Sendai, 1985, 11-40, Adv. Stud. Pure Math., 10, North-Holland, Amsterdam, 1987.

[27] V. Bangert and Y. M. Long, The existense of two closed geodesics on every Finsler 2-sphere. Preprint (2005). Submitted.

[28] R. Bartnik, Existence of maximal surfaces in asymptotically flat spacetimes. Comm. Math. Phys. 94 (1984), no. 2, 155-175.

[29] D. Bao, S.-S. Chern and Z. Shen, An introduction to Riemann-Finsler geometry. Graduate Texts in Mathematics, 200. Springer-Verlag, New York, 2000.

[30] R. Bartnik, Quasi-spherical metrics and prescribed scalar curvature. J. Differential Geom. 37(1993), no. 1, 31-71.

[31] R. Bartnik and J. McKinnon, Particlelike solutions of the Einstein-Yang-Mills equations. Phys. Rev. Lett. 61(1988), no. 2, 141-144.

[32] P. de Bartolomeis and G. Tian, Stability of complex vector bundles. J. Differential Geom. 43(1996), no. 2, 231-275.

[33] A. Beauville, Variétés Kähleriennes dont la première classe de Chern est nulle. J. Differential Geom. 18(1983), no. 4, 755-782 (1984).

[34] K. Becker and K. Dasgupta, Heterotic strings with torsion. J. High Energy Phys. 11(2002), 006, $30 \mathrm{pp}$.

[35] M. Berger, Sur les groupes d'holonomie homogène des variétés à connexion affine et des variétés riemanniennes. Bull. Soc. Math. France 83 (1955), 279-330.

[36] M. Berger, Sur quelques variétés d'Einstein compactes. Ann. Mat. Pura Appl. (4) 53(1961),89-95.

[37] M. Bershadsky, S. Cecotti, H. Ooguri and C. Vafa, Kodaira-Spencer theory of gravity and exact results for quantum string amplitudes. Comm. Math. Phys. 165(1994), no. 2, 311-427.

[38] G. Besson, G. Courtois and S. Gallot, Entropies et rigidités des espaces localement symétriques de courbure strictement négative. Geom. Funct. Anal. 5(1995), no. 5, 731799.

[39] J. M. Bismut, The Atiyah-Singer index theorem for families of Dirac operators: two heat equation proofs. Invent. Math. 83(1986), no. 1, 91-151.

[40] J. M. Bismut and S. Goette, Equivariant de Rham torsions. Ann. of Math. (2) 159(2004), no. $1,53-216$.

[41] J. M. Bismut and W. P. Zhang, An extension of a theorem by Cheeger and Müller. With an appendix by François Laudenbach. Astérisque No. 205 (1992), 235 pp.

[42] J. M. Bismut and W. P. Zhang, Milnor and Ray-Singer metrics on the equivariant determinant of a flat vector bundle. Geom. Funct. Anal. 4(1994), no. 2, 136-212.

[43] S. Bochner, Curvature and Betti numbers. Ann. of Math. (2) 49(1948). 379-390.

[44] F. A. Bogomolov, Hamiltonian Kälerian manifolds. Dokl. Akad. Nauk SSSR 243(1978), no. $5,1101-1104$. 
[45] E. Bombieri and E. Giusti, Harnack's inequality for elliptic differential equations on minimal surfaces. Invent. Math. 15(1972), 24-46.

[46] E. Bombieri, De Giorgi E. and E. Giusti, Minimal cones and the Bernstein problem. Invent. Math. 7(1969), 243-268.

[47] R. E. Borcherds, Automorphic forms on $\mathrm{O}_{s+2,2}(R)$ and infinite products. Invent. Math. 120(1995), no. 1, 161-213.

[48] R. E. Borcherds, The moduli space of Enriques surfaces and the fake Monster Lie superalgebra. Topology 35(1996), no. 3, 699-710.

[49] R. Bott, A residue formula for holomorphic vector-fields. J. Differential Geometry 1(1967), 311-330.

[50] R. Bott and C. H. Taubes, Clifford On the rigidity theorems of Witten. J. Amer. Math. Soc. 2(1989), no. 1, 137-186.

[51] L. Boutet de Monvel and J. Sjöstrand, Sur la singularité des noyaux de Bergman et de Szegö. Journées: Équations aux Dérivées Partielles de Rennes (1975), Asterisque 34-35, Soc. Math. France, Paris, 1976, 123-164.

[52] C. P. Boyer, K. Galicki and J. Kollár, Einstein Metrics on Spheresmath. arXiv:math.DG/0309408.

[53] P. J. Braam and S. K. Donaldson, Floer's work on instanton homology, knots and surgery. The Floer memorial volume, 195-256, Progr. Math., 133, Birkhäuser, Basel, 1995.

[54] K. A. Brakke, The motion of a surface by its mean curvatur. Mathematical Notes, 20. Princeton University Press, Princeton, N.J., 1978.

[55] H. L. Bray, Proof of the Riemannian Penrose inequality using the positive mass theorem. J. Differential Geom. 59(2001), no. 2, 177-267.

[56] J. D. Brown and J. W. York, Quasilocal energy and conserved charges derived from the gravitational action. Phys. Rev. D (3) 47(1993), no. 4, 1407-1419.

[57] R. Bryant, Conformal and minimal immersions of compact surfaces into the 4-sphere. J. Differential Geom. 17(1982), no. 3, 455-473.

[58] R. L. Bryant, Metrics with exceptional holonomy. Ann. of Math. (2) 126(1987), no. 3, $525-576$.

[59] R. Bryant and S. Salamon, On the construction of some complete metrics with exceptional holonomy, Duke Math. J. 58(1989), 829-850.

[60] N. P. Buchdahl, Hermitian-Einstein connections and stable vector bundles over compact complex surfaces. Math. Ann. 280(1988), no. 4, 625-648.

[61] Y. Burago, M. Gromov and G. A. D. Perelman, Aleksandrov spaces with curvatures bounded below. Uspekhi Mat. Nauk 47(1992), no. 2(284), 3-51, 222; translation in Russian Math. Surveys 47(1992), no. 2, 1-58.

[62] F. E. Burstall, D. Ferus, F. Pedit, U. Pinkall, Harmonic tori in symmetric spaces and commuting Hamiltonian systems on loop algebras. Ann. of Math. (2) 138(1993), no. 1, $173-212$.

[63] A. Butscher, Regularizing a singular special Lagrangian variety. Comm. Anal. Geom. 12(2004), no. 4, 733-791.

[64] L. Caffarelli, R. Hardt and L. Simon, Minimal surfaces with isolated singularities. Manuscripta Math. 48(1984), no. 1-3, 1-18.

[65] L. Caffarelli, L. Nirenberg and J. Spruck, The Dirichlet problem for nonlinear second order elliptic equations, III. Functions of the eigenvalues of the Hessian. Acta Math. 155(1985), 261-301.

[66] E. Calabi, On Kähler manifolds with vanishing canonical class. Algebraic geometry and topology. A symposium in honor of S. Lefschetz, pp. 78-89. Princeton University Press, Princeton, N. J., 1957.

[67] E. Calabi, Improper affine hyperspheres of convex type and a generalization of a theorem by K. Jörgens. Michigan Math. J. 5 (1958) 105-126. 
[68] E. Calabi and E. Vesentini, Sur les variétés complexes compactes localement symétriques. Bull. Soc. Math. France 87(1959) 311-317.

[69] P. Candelas, G. T. Horowitz, A. Strominger and E. Witten, Vacuum configurations for superstrings. Nuclear Phys. B 258(1985), no. 1, 46-74.

[70] P. Candelas, de la Ossa, X. C., P. S. Green and L. Parkes, A pair of Calabi-Yau manifolds as an exactly soluble superconformal theory. Nuclear Phys. B 359(1991), no. 1, 21-74.

[71] H. D. Cao, Deformation of Kähler metrics to Kähler-Einstein metrics on compact Kähler manifolds. Invent. Math. 81(1985), no. 2, 359-372.

[72] H.-D. Cao, On Harnack inequalities for the Kähler-Ricci flow. Invent. Math. 109(1992), $247-263$.

[73] H. D. Cao, Limits of solutions to the Khler-Ricci flow. J. Differential Geom. 45(1997), no. $2,257-272$.

[74] J. G. Cao and J. F. Escobar, A new 3-dimensional curvature integral formula for PLmanifolds of non-positive curvature. Comm. Anal. Geom. 11(2003), no. 3, 489-551.

[75] J. G. Cao, H. J. Fan and F. Ledrappier, Martin points on open manifolds of non-positive curvature. To appear in Trans. AMS.

[76] G. L. Cardoso, G. Curio, G. Dall'Agata and D. Lüst, Heterotic string theory on nonKähler manifolds with $H$-flux and gaugino condensate. Proceedings of the 36th International Symposium Ahrenshoop on the Theory of Elementary Particles. Fortschr. Phys. 52(2004), no. 6-7, 483-488.

[77] D. Catlin, The Bergman kernel and a theorem of Tian. Analysis and geometry in several complex variables (Katata, 1997), 1-23, Trends Math., Birkhäuser Boston, Boston, MA, 1999.

[78] S. C. Chang, Recent developments on the Calabi flow. Geometric evolution equations, 17-42, Contemp. Math., 367, Amer. Math. Soc., Providence, RI, 2005.

[79] S. Y. A. Chang, M. J. Gursky and P. Yang, An a priori estimate for a fully nonlinear equation on four-manifolds. Dedicated to the memory of Thomas H. Wolff. J. Anal. Math. 87(2002), 151-186.

[80] A. Chau and L.-F. Tam, On the complex structure of Kähler manifolds with nonnegative curvature. arXiv:math.DG/0504422.

[81] J. Cheeger, A lower bound for the smallest eigenvalue of the Laplacian. Problems in analysis (Papers dedicated to Salomon Bochner, 1969), pp. 195-199. Princeton Univ. Press, Princeton, N. J., 1970.

[82] J. Cheeger, Analytic torsion and Reidemeister torsion. Proc. Nat. Acad. Sci. U.S.A. 74(1977), no. 7, 2651-2654.

[83] J. Cheeger and T. H. Colding, On the structure of spaces with Ricci curvature bounded below. I. J. Differential Geom. 46(1997), no. 3, 406-480.

[84] J. Cheeger and T. H. Colding, On the structure of spaces with Ricci curvature bounded below. II. J. Differential Geom. 54(2000), no. 1, 13-35.

[85] J. Cheeger and T. H. Colding, On the structure of spaces with Ricci curvature bounded below. III. J. Differential Geom. 54(2000), no. 1, 37-74.

[86] J. Cheeger and D. Gromoll, The splitting theorem for manifolds of nonnegative Ricci curvature. J. Differential Geometry 6(1971/72), 119-128.

[87] B. L. Chen, S. H. Tang and X. P. Zhu, A uniformization theorem for complete non-compact Kähler surfaces with positive bisectional curvature. J. Differential Geom. 67(2004), no. 3, 519-570.

[88] B. L. Chen and X. P. Zhu, Complete Riemannian manifolds with pointwise pinched curvature. Invent. Math. 140(2000), no. 2, 423-452

[89] B. L. Chen and Z. X. Ping, On complete noncompact Kähler manifolds with positive bisectional curvature. Math. Ann. 327 (2003), no. 1, 1-23.

[90] B. L. Chen and X. P. Zhu, Volume growth and curvature decay of positive curved Kähler manifold, arXiv:math.DG/0211374. 
[91] B. L. Chen and X. P. Zhu, Flow with Surgery on Four-manifolds with Positive Isotropic Curvature. arXiv:math.DG/0504478.

[92] B. L. Chen and X. P. Zhu, Uniqueness of the Ricci Flow on Complete Noncompact Manifolds. arXiv:math.DG/0505447.

[93] C. C. Chen and C. S. Lin, A spherical Harnack inequality for singular solutions of nonlinear elliptic equations. Ann. Scuola Norm. Sup. Pisa Cl. Sci. (4) 30(2001), no. 3-4, 713-738 (2002).

[94] M. F. Chen and F. Y. Wang, Application of coupling method to the first eigenvalue on manifold. Progr. Natur. Sci. (English Ed.) 5(1995), no. 2, 227-229.

[95] X. X. Chen, The space of Kähler metrics. J. Differential Geom. 56(2000), no. 2, 189-234.

[96] Y. G. Chen, Y. Giga and S. Goto, Uniqueness and existence of viscosity solutions of generalized mean curvature flow equations. J. Differential Geom. 33(1991), no. 3, 749786.

[97] S. Y. Cheng, Eigenvalue comparison theorems and its geometric applications. Math. Z. 143(1975), no. 3, 289-297.

[98] S. Y. Cheng, Eigenfunctions and nodal sets. Comment. Math. Helv. 51(1976), no. 1, $43-55$.

[99] S. Y. Cheng, P. Li and S.-T. Yau, On the upper estimate of the heat kernel of a complete Riemannian manifold. Amer. J. Math. 103(1981), no. 5, 1021-1063.

[100] S. Y. Cheng, P. Li and S.-T. Yau, Heat equations on minimal submanifolds and their applications. Amer. J. Math. 106(1984), no. 5, 1033-1065.

[101] S. Y. Cheng and S.-T. Yau, Differential equations on Riemannian manifolds and their geometric applications, Comm. Pure Appl. Math. 28(1975), no. 3, 333-354.

[102] S. Y. Cheng and S.-T. Yau, Maximal space-like hypersurfaces in the Lorentz-Minkowski spaces. Ann. of Math. (2) 104(1976), no. 3, 407-419.

[103] S. Y. Cheng and S. T. Yau, On the regularity of the solution of the $\mathrm{n}$-dimensional Minkowski problem. Commun. Pure Appl. Math. 29(1976), 495-516.

[104] S. Y. Cheng and S.-T. Yau, On the existence of a complete Kähler metric on noncompact complex manifolds and the regularity of Fefferman's equation. Comm. Pure Appl. Math. 33(1980), no. 4, 507-544.

[105] S. Y. Cheng and S.-Y. Yau, The real Monge-Ampère equation and affine flat structures, in Proceedings of the 1980 Beijing Symposium on Differential Geometry and Differential Equations, Science Press, Beijing, China (1982), Gordon and Breach Science Publishing Company, New York, pp. 339-370.

[106] S. Y. Cheng and S. T. Yau, Complete affine hypersurfaces. I. The completeness of affine metrics. Comm. Pure Appl. Math. 39(1986), no. 6, 839-866.

[107] Proceedings of the 1980 Beijing Symposium on Differential Geometry and Differential Equations. Vol. 1, 2, 3. Held in Beijing, August 18-September 21, 1980. Edited by S. S. Chern and Wen Tsün Wu [Wen Jun Wu]. Science Press, Beijing; Gordon and Breach Science Publishers, New York, 1982.

[108] S. S. Chern, Characteristic classes of Hermitian manifolds. Ann. of Math. (2) 47(1946). $85-121$.

[109] S. S. Chern and J. Simons, Characteristic forms and geometric invariants. Ann. of Math. (2) 99(1974), 48-69.

[110] H. I. Choi and R. Schoen, The space of minimal embeddings of a surface into a threedimensional manifold of positive Ricci curvature. Invent. Math. 81(1985), no. 3, 387-394.

[111] H. I. Choi and A. Treibergs, Gauss maps of spacelike constant mean curvature hypersurfaces of Minkowski space. J. Differential Geom. 32(1990), no. 3, 775-817.

[112] H. I. Choi and A. N. Wang, A first eigenvalue estimate for minimal hypersurfaces. J. Differential Geom. 18(1983), no. 3, 559-562.

[113] S. Y. Choi and W. M. Goldman, The classification of real projective structures on compact surfaces. Bull. Amer. Math. Soc. (N.S.) 34(1997), no. 2, 161-171. 
[114] Y. Choquet-Bruhat and V. Moncrief, Future global in time Einsteinian spacetimes with U(1) isometry group. Ann. Henri Poincare 2(2001), no. 6, 1007-1064.

[115] Y. Choquet-Bruhat and J. W. York, On H. Friedrich's formulation of the Einstein equations with fluid sources. Topol. Methods Nonlinear Anal. 18(2001), no. 2, 321-335.

[116] D. Christodoulou, The instability of naked singularities in the gravitational collapse of a scalar field. Ann. of Math. (2) 149(1999), no. 1, 183-217.

[117] D. Christodoulou and S. Klainerman, The global nonlinear stability of the Minkowski space. Princeton Mathematical Series, 41. Princeton University Press, Princeton, NJ, 1993.

[118] D. Christodoulou and A. Tahvildar-Zadeh, On the regularity of spherically symmetric wave maps. Comm. Pure Appl. Math. 46(1993), no. 7, 1041-1091.

[119] D. Christodoulou and S.-T. Yau, Some remarks on the quasi-local mass. Mathematics and general relativity, 9-14, Contemp. Math., 71, Amer. Math. Soc., Providence, RI, 1988.

[120] P. T. Chruúsciel, Semi-global existence and convergence of solutions of the RobinsonTrautman (2-dimensional Calabi) equation. Comm. Math. Phys. 137(1991), no. 2, 289313.

[121] F. Chung, Discrete Isoperimetric Inequalities, Surveys in Differential Geometry, IX, Inrernational Press, pp.53-82.

[122] C. H. Clemens, Double solids. Adv. in Math. 47(1983), no. 2, 107-230.

[123] T. H. Colding, Shape of manifolds with positive Ricci curvature. Invent. Math. 124(1996), no. 1-3, 175-191.

[124] T. H. Colding and W. P. Minicozzi II, Harmonic functions on manifolds. Ann. of Math. (2) 146(1997), no. 3, 725-747.

[125] T. H. Colding, and W. P. Minicozzi II, Embedded minimal surfaces without area bounds in 3-manifolds. Geometry and topology: Aarhus (1998), 107-120, Contemp. Math., 258, Amer. Math. Soc., Providence, RI, 2000.

[126] T. H. Colding and W. P. Minicozzi II The space of embedded minimal surfaces of fixed genus in a 3-manifold. I. Estimates off the axis for disks. Ann. of Math. (2) 160 (2004), no. $1,27-68$.

[127] T. H. Colding and W. P. Minicozzi II The space of embedded minimal surfaces of fixed genus in a 3-manifold. II. Multi-valued graphs in disks. Ann. of Math. (2) 160 (2004), no. 1, 69-92.

[128] T. H. Colding and W. P. Minicozzi II The space of embedded minimal surfaces of fixed genus in a 3-manifold. III. Planar domains. Ann. of Math. (2) 160 (2004), no. 2 , $523-572$

[129] T. H. Colding and W. P. Minicozzi II The space of embedded minimal surfaces of fixed genus in a 3-manifold. IV. Locally simply connected. Ann. of Math. (2) 160(2004), no. 2, 573-615.

[130] T. H. Colding and W. P. Minicozzi II, Estimates for the extinction time for the Ricci flow on certain 3-manifolds and a question of Perelman. J. Amer. Math. Soc. 18(2005), no. $3,561-569$.

[131] T. H. Colding and W. P. Minicozzi II, An excursion into geomtric analysis. Surveys in Differential Geomtry IX, International Press, 83-146.

[132] Colin de Verdière, Y. Sur la multiplicitë de la première valeur propre non nulle du laplacien. Comment. Math. Helv. 61(1986), no. 2, 254-270.

[133] K. Corlette, Archimedean superrigidity and hyperbolic geometry. Ann. of Math. (2) 135(1992), no. 1, 165-182.

[134] C. B. Croke, Some isoperimetric inequalities and eigenvalue estimates. Ann. Sci. ecole Norm. Sup. (4) 13(1980), no. 4, 419-435.

[135] C. B. Croke, A sharp four-dimensional isoperimetric inequality. Comment. Math. Helv. 59(1984), no. 2, 187-192. 
[136] M. Dafermos, Stability and instability of the Cauchy horizon for the spherically symmetric Einstein-Maxwell-scalar field equations. Ann. of Math. (2) 158(2003), no. 3, 875-928.

[137] X. Z. Dai, X. D. Wang and G. F. Wei, On the stability of Riemannian manifold with parallel spinors. Invent. Math. 161(2005), 151 - 176.

[138] G. D. Daskalopoulos and R. A. Wentworth, Geometric quantization for the moduli space of vector bundles with parabolic structure. Geometry, topology and physics, 119-155, de Gruyter, Berlin, 1997.

[139] J. Demailly, Holomorphic Morse inequalities. Several complex variables and complex geometry. 93-114, Proc. Sympos. Pure Math., 52, Part 2, Amer. Math. Soc., Providence, RI, 1991.

[140] J. Demailly, A numerical criterion for very ample line bundles. J. Differential Geom. 37(1993), no. 2, 323-374.

[141] J. Demailly, Effective bounds for very ample line bundles. Invent. Math. 124(1996), no. $1-3,243-261$

[142] R. Dijkgraaf, H. Verlinde and E. Verlinde, Topological strings in $d<1$. Nuclear Phys. B 352(1991), no. 1, 59-8.

[143] S. K. Donaldson, An application of gauge theory to four-dimensional topology. J. Differential Geom. 18(1983), no. 2, 279-315.

[144] S. K. Donaldson, Anti self-dual Yang-Mills connections over complex algebraic surfaces and stable vector bundles. Proc. London Math. Soc. (3) 50(1985), no. 1, 1-26.

[145] S. K. Donaldson, Infinite determinants, stable bundles and curvature. Duke Math. J. 54(1987), no. 1, 231-247.

[146] S. K. Donaldson, The Seiberg-Witten equations and 4-manifold topology. Bull. Amer. Math. Soc. (N.S.) 33(1996), no. 1, 45-70.

[147] S. K. Donaldson, Symplectic submanifolds and almost-complex geometry. J. Differential Geom. 44(1996), no. 4, 666-705.

[148] S. K. Donaldson, Lefschetz pencils on symplectic manifolds. J. Differential Geom. 53(1999), no. 2, 205-236.

[149] S. K. Donaldson, Scalar curvature and projective embeddings. I. J. Differential Geom. 59(2001), no. 3, 479-522.

[150] S. K. Donaldson, Scalar curvature and stability of toric varieties. J. Differential Geom. 62(2002), no. 2, 289-349.

[151] S. K. Donaldson and R. D. Friedman, Connected Sums of Self-Dual Manifolds and Deformations of Singular Spaces. Nonlinearity 2(1989), 197-239.

[152] R. T. Dong, Nodal sets of eigenfunctions on Riemann surfaces. J. Differential Geom. 36(1992), no. 2, 493-506.

[153] H. Donnelly and C. Fefferman, Nodal sets of eigenfunctions on Riemannian manifolds. Invent. Math. 93(1988), no. 1, 161-183.

[154] J. J. Duistermaat and L. Hörmander, Fourier integral operators. II. Acta Math. 128(1972), no. 3-4, 183-269.

[155] J. J. Duistermaat and V. W. Guillemin, The spectrum of positive elliptic operators and periodic bicharacteristics. Invent. Math. 29(1975), no. 1, 39-79.

[156] J. Eells Jr. and J. H. Sampson, Harmonic mappings of Riemannian manifolds. Amer. J. Math. 86(1964), 109-160.

[157] L. Ein and R. Lazarsfeld, Global generation of pluricanonical and adjoint linear series on smooth projective threefolds. J. Amer. Math. Soc. 6(1993), no. 4, 875-903.

[158] L. C. Evans and J. Spruck, Motion of level sets by mean curvature. I. J. Differential Geom. 33(1991), no. 3, 635-681.

[159] H. Federer and W. H. Fleming, Normal and integral currents. Ann. of Math. (2) $\mathbf{7 2}(1960), 458-520$.

[160] C. Fefferman, The Bergman kernel and biholomorphic mappings of pseudoconvex domains. Invent. Math. 26(1974), 1-65. 
[161] C. Fefferman and D. H. Phong, The uncertainty principle and sharp Gärding inequalities. Comm. Pure Appl. Math. 34(1981), no. 3, 285-331.

[162] F. Finster, N. Kamran, J. Smoller and S.-T. Yau, Decay rates and probability estimates for massive Dirac particles in the Kerr-Newman black hole geometry. Comm. Math. Phys. 230(2002), no. 2, 201-244.

[163] D. Fischer-Colbrie and R. Schoen, The structure of complete stable minimal surfaces in 3-manifolds of nonnegative scalar curvature. Comm. Pure Appl. Math. 33(1980), no. 2, $199-211$.

[164] A. Floer, An instanton-invariant for 3-manifolds. Comm. Math. Phys. 118(1988), no. $2,215-240$.

[165] A. Floer, Morse theory for Lagrangian intersections. J. Differential Geom. 28(1988), no. $3,513-547$.

[166] A. Floer, Witten's complex and infinite-dimensional Morse theory. J. Differential Geom. 30(1989), no. 1, 207-221.

[167] D. S. Freed, Characteristic numbers and generalized path integrals. Geometry, topology and physics, 126-138, Conf. Proc. Lecture Notes Geom. Topology, IV, Internat. Press, Cambridge, MA, 1995.

[168] M. Freedman, The topology of four-dimensional manifolds. J. Differential Geom. 17(1982), no. 3, 357-453.

[169] M. Freedman, J. Hass and P. Scott, Least area incompressible surfaces in 3-manifolds. Invent. Math. 71(1983), no. 3, 609-642.

[170] A. Freire, On the Martin boundary of Riemannian products. J. Differential Geom. 33(1991), no. 1, 215-232.

[171] R. Friedman, Simultaneous resolution of threefold double points. Math. Ann. 274(1986), no. 4, 671-689.

[172] H. Friedrich, Hyperbolic reductions for Einstein's equations. Classical Quantum Gravity. 13(1996), no. 6, 1451-1469.

[173] J. X. Fu and S.-T. Yau, Existence of Supersymmetric Hermitian Metrics with Torsion on Non-Kaehler Manifolds. arXiv:hep-th/0509028.

[174] T. Fujita, On polarized manifolds whose adjoint bundles are not semipositive. Algebraic geometry, Sendai, 1985, 167-178, Adv. Stud. Pure Math., 10, North-Holland, Amsterdam, 1987.

[175] K. Fukaya, Morse homotopy, $A^{\infty}$-category, and Floer homologies. Proceedings of GARC Workshop on Geometry and Topology '93 (Seoul, 1993), 1-102, Lecture Notes Ser., 18, Seoul Nat. Univ., Seoul, 1993.

[176] K. Fukaya and Y. G. Oh, Zero-loop open strings in the cotangent bundle and Morse homotopy. Asian J. Math. 1(1997), no. 1, 96-180.

[177] K. Fukaya and K. Ono, Arnold conjecture and Gromov-Witten invariant. Topology 38(1999), no. 5, 933-1048.

[178] A. Futaki, An obstruction to the existence of Einstein-K?hler metrics. Inv. Math., 73(1983), 437-443.

[179] H. Garland, p-adic curvature and the ohomology of discrete subgroups of p-adic groups. Ann. of Math. (2) 97(1973), 375-423.

[180] J. P. Gauntlett, D. Martelli, J. Sparks and D. Waldram, Sasaki-Einstein metrics on $S^{2} \times S^{3}$. Adv. Theor. Math. Phys. 8(2004), no. 4, 711-734.

[181] C. Gerhardt, H-surfaces in Lorentzian manifolds. Comm. Math. Phys. 89(1983), no. 4 , $523-553$

[182] C. Gerhardt, Hypersurfaces of prescribed scalar curvature in Lorentzian manifolds. J. Reine Angew. Math. 554(2003), 157-199.

[183] R. Geroch, Energy extraction. Ann. New York Acad. Sci., 224, 108-117.

[184] M. L. Gerver and N. S. Nadirashvili, A condition for isometry of Riemannian metrics in the disc. Dokl. Akad. Nauk SSSR 275(1984), no. 2, 289-293. 
[185] D.Gieseker, On moduli of vector bundles on an algebraic surface, Ann. of Math. 106(1977) 45-60.

[186] A. B. Givental, Equivariant Gromov-Witten invariants. Internat. Math. Res. Notices 1996, no. 13, 613-663.

[187] W. M. Goldman, On the polynomial cohomology of affine manifolds. Invent. Math. 65(1981/82), no. 3, 453-457.

[188] R. E. Gompf, Toward a topological characterization of symplectic manifolds. J. Symplectic Geom. 2(2004), no. 2, 177-206.

[189] C. Gordon, D. Webb and S. Wolpert, Isospectral plane domains and surfaces via Riemannian orbifolds. Invent. Math. 110(1992), no. 1, 1-22.

[190] M. Goresky, G. Harder and R. MacPherson, Weighted cohomology. Invent. Math. 116(1994), no. 1-3, 139-213.

[191] A. Gray, Invariants of curvature operators of four-dimensional Riemannian manifolds. Proceedings of the Thirteenth Biennial Seminar of the Canadian Mathematical Congress Vol. 2, pp. 42-65. Canad. Math. Congr., Montreal, Que., 1972.

[192] R. E. Greene and H. Wu, Analysis on noncompact Kähler manifolds. Proc. Sympos. Pure Math., Vol. XXX, Part 2, 69-100. Amer. Math. Soc., Providence, R.I., 1977.

[193] A. Grigor'yan, Heat kernel of a noncompact Riemannian manifold. Proc. Sympos. Pure Math., 57, 239-263. Amer. Math. Soc., Providence, RI, 1995.

[194] A. Grigor'yan, Y. Netrusov and S.-T. Yau, Eigenvalues of elliptic operators and geometric applications, in Servey in Differential Geometry, V. IX, 147-217. A. Grigor'yan and S.-T. Yau ed., International Press.

[195] A. Grigor'yan and L. Saloff-Coste, Dirichlet heat kernel in the exterior of a compact set. Comm. Pure Appl. Math. 55(2002), no. 1, 93-133.

[196] D. Gromoll and W. Meyer, On complete open manifolds of positive curvature. Ann. of Math. (2) 90(1969) 75-90.

[197] D. Gromoll and W. Meyer, Periodic geodesics on compact riemannian manifolds. J. Differential Geometry 3(1969), 493-510.

[198] D. Gromoll and J. A. Wolf, Some relations between the metric structure and the algebraic structure of the fundamental group in manifolds of nonpositive curvature. Bull. Amer. Math. Soc. 77(1971), 545-552.

[199] M. Gromov, Manifolds of negative curvature. J. Differential Geom. 13(1978), no. 2, 223-230.

[200] M. C. Gromov, Curvature, diameter and Betti numbers. Comment. Math. Helv. 56(1981), no. 2, 179-195.

[201] M. Gromov, Volume and bounded cohomology. Inst. Hautes Études Sci. Publ. Math. 56 (1982), 5-99.

[202] M. Gromov, Pseudoholomorphic curves in symplectic manifolds. Invent. Math. 82(1985), no. $2,307-347$.

[203] M. Gromov, Metric structures for Riemannian and non-Riemannian spaces. Based on the 1981 French original. With appendices by M. Katz, P. Pansu and S. Semmes. Translated from the French by Sean Michael Bates. Progress in Mathematics, 152. Birkhäuser Boston, Inc., Boston, MA, 1999.

[204] M. Gromov and H. B. Lawson Jr., Spin and scalar curvature in the presence of a fundamental group. I. Ann. of Math. (2) 111(1980), no. 2, 209-230.

[205] M. Gromov and H. B. Lawson Jr., The classification of simply connected manifolds of positive scalar curvature. Ann. of Math. (2) 111(1980), no. 3, 423-434.

[206] M. Gromov and R. Schoen, Harmonic maps into singular spaces and p-adic superrigidity for lattices in groups of rank one. Inst. Hautes tudes Sci. Publ. Math. 76(1992), 165-246.

[207] K. Grove and K. Shiohama, A generalized sphere theorem. Ann. Math. (2) 106(1977), no. 2, 201-211.

[208] C. H. Gu, On the initial-boundary value problem for harmonic maps from the 2dimensional Minkowski space. Manuscripta Math. 33(1980/81), no. 1, 51-58. 
[209] B. Guan and P. Guan, Hypersurfaces with prescribed curvatures. Ann. Math. 156(2002), 655-674.

[210] P. F. Guan and X. N. Ma, The Christoffel-Minkowski problem. I. Convexity of solutions of a Hessian equation. Invent. Math. 151(2003), no. 3, 553-577.

[211] V. Guillemin, Wave-trace invariants. Duke Math. J. 83(1996), no. 2, 287-352.

[212] S. Gukov, Three-dimensional quantum gravity, Chern-Simons theory, and the A-polynomial. Comm. Math. Phys. 255(2005), no. 3, 577-627.

[213] R. Gulliver, Regularity of minimizing surfaces of prescribed mean curvature. Ann. of Math. (2) 97(1973), 275-305.

[214] W. Haken, Various aspects of the three-dimensional Poincarè problem. 1970 Topology of Manifolds (Proc. Inst., Univ. of Georgia, Athens, Ga., 1969) pp. 140-152 Markham, Chicago, Ill .

[215] R. S. Hamilton, Three-manifolds with positive Ricci curvature. J. Differential Geom. 17(1982), no. 2, 255-306.

[216] R. S. Hamilton, The Ricci flow on surfaces. Mathematics and general relativity, 237-262, Contemp. Math., 71, Amer. Math. Soc., Providence, RI, 1988.

[217] R. S. Hamilton, The Harnack estimate for the Ricci flow. J. Differential Geom. 37(1993), no. $1,225-243$

[218] R. S. Hamilton, Four-manifolds with positive isotropic curvature. Comm. Anal. Geom. 5(1997), no. 1, 1-92.

[219] Q. Han, R. Hardt and F. H. Lin, Geometric measure of singular sets of elliptic equations. Comm. Pure Appl. Math. 51(1998), no. 11-12, 1425-1443.

[220] Q. Han, J. X. Hong and C. S. Lin, Local isometric embedding of surfaces with nonpositive Gaussian curvature. J. Differential Geom. 63(2003), no. 3, 475-520.

[221] R. Hardt and L. Simon, Boundary regularity and embedded solutions for the oriented Plateau problem. Ann. of Math. (2) 110(1979), no. 3, 439-486.

[222] F. R. Harvey and H. B. Lawson Jr., On boundaries of complex analytic varieties. I. Ann. of Math. (2) 102(1975), no. 2, 223-290.

[223] F. R. Harvey and H. B. Lawson Jr., On boundaries of complex analytic varieties. II. Ann. Math. (2) 106(1977), no. 2, 213-238.

[224] R. Harvey and H. B. Lawson, Jr. Calibrated geometries. Acta Math. 148(1982), 47-157.

[225] A. Hassell, R. Melrose and A. Vasy, Spectral and scattering theory for symbolic potentials of order zero. Adv. Math. 181(2004), no. 1, 1-87.

[226] J. Hersch, Quatre propriétés isopérimétriques de membranes sphériques homogènes. C. R. Acad. Sci. Paris Sér. A-B 270(1970), A1645-A1648.

[227] N. Hingston, Equivariant Morse theory and closed geodesics. J. Differential Geom. 19(1984), no. $1,85-116$.

[228] F. Hirzebruch and K. Kodaira, On the complex projective spaces. J. Math. Pures Appl. (9) 36(1957), 201-216.

[229] N. Hitchin, Harmonic spinors. Advances in Math. 14(1974), 1-55.

[230] N. Hitchin, Compact four-dimensional Einstein manifolds. J. Differential Geometry 9(1974), 435-441.

[231] N. J. Hitchin, The self-duality equations on a Riemann surface. Proc. London Math. Soc. (3) 5(1987), no. 1, 59-126.

[232] N. J. Hitchin, Harmonic maps from a 2-torus to the 3-sphere. J. Differential Geom. 31(1990), no. 3, 627-710.

[233] N. J. Hitchin, Lie groups and Teichmuller space. Topology 31(1992), no. 3, 449-473.

[234] N. J. Hitchin, The moduli space of special Lagrangian submanifolds. Ann. Scuola Norm. Sup. Pisa Cl. Sci. (4) 25(1997), no. 3-4, 503-515.

[235] N. Hitchin, The geometry of three-forms in six dimensions. J. Differential Geom. $\mathbf{5 5}(2000)$, no. $3,547-576$. 
[236] N. Hitchin, Stable forms and special metrics. Global differential geometry: the mathematical legacy of Alfred Gray (Bilbao, 2000), 70-89, Contemp. Math., 288 , Amer. Math. Soc., Providence, RI, 2001.

[237] H. Hofer, K. Wysocki and E. Zehnder, The dynamics on three-dimensional strictly convex energy surfaces. Ann. of Math. (2) 148(1998), no. 1, 197-289.

[238] M. Hoffmann-Ostenhof, T. Hoffmann-Ostenhof and N. Nadirashvili, Critical sets of smooth solutions to elliptic equations in dimension 3, Indiana Univ. Math. J. 45(1996), $15-37$.

[239] J. X. Hong, Realization in $R^{3}$ of complete Riemannian manifolds with negative curvature. Comm. Anal. Geom. 1(1993), no. 3-4, 487-514.

[240] M. J. Hopkins, Topological modular forms, the Witten genus, and the theorem of the cube. Proceedings of the International Congress of Mathematicians, Vol. 1, 2 (Zürich, 1994), 554-565, Birkhäuser, Basel, 1995.

[241] L. Hörmander, An introduction to complex analysis in several variables. Third edition. North-Holland Mathematical Library, 7. North-Holland Publishing Co., Amsterdam, 1990.

[242] L.K. Hua, Harmonic analysis of functions of several variables in the classical domains. American Mathematical Society, Providence, RI, 1963.

[243] G. Huisken, Flow by mean curvature of convex surfaces into spheres. J. Differential Geom. 20(1984), no. 1, 237-266.

[244] G. Huisken, Contracting convex hypersurfaces in Riemannian manifolds by their mean curvature. Invent. Math. 84(1986), no. 3, 463-480.

[245] G. Huisken and T. Ilmanen, The inverse mean curvature flow and the Riemannian Penrose inequality. J. Differential Geom. 59(2001), no. 3, 353-437.

[246] G. Huisken and C. Sinestrari, Mean curvature flow singularities for mean convex surfaces. Calc. Var. Partial Differential Equations 8(1999), no. 1, 1-14.

[247] G. Huisken and C. Sinestrari, Convexity estimates for mean curvature flow and singularities of mean convex surfaces. Acta Math. 183(1999), no. 1, 45-70.

[248] G. Huisken and S.-T. Yau, Definition of center of mass for isolated physical systems and unique foliations by stable spheres with constant mean curvature. Invent. Math. 124(1996), no. 1-3, 281-311.

[249] H. Iwaniec and P. Sarnak, $L^{\infty}$ norms of eigenfunctions of arithmetic surfaces. Ann. of Math. (2) 141(1995), no. 2, 301-320.

[250] L. Z. Ji and R. MacPherson, Geometry of compactifications of locally symmetric spaces. Ann. Inst. Fourier (Grenoble) 52(2002), no. 2, 457-559.

[251] M. Ji and G. Y. Wang, Minimal surfaces in Riemannian manifolds. Mem. Amer. Math. Soc. 104(1993), no. 495.

[252] K. Jörgens, Über die Losungen der Differentialgleichung $r t-s^{2}=1$. Math. Ann. $\mathbf{1 2 7}(1954), 130-134$.

[253] J. Jorgenson and A. Todorov, Enriques surfaces, analytic discriminants, and Borcherds's $\Phi$ function. Comm. Math. Phys. 191(1998), no. 2, 249-264.

[254] J. Jost, Embedded minimal surfaces in manifolds diffeomorphic to the three-dimensional ball or sphere. J. Differential Geom. 30(1989), no. 2, 555-577.

[255] J. Jost, Convex functionals and generalized harmonic maps into spaces of nonpositive curvature. Comment. Math. Helv. 70(1995), no. 4, 659-673.

[256] J. Jost, and S.-T. Yau, Applications of quasilinear PDE to algebraic geometry and arithmetic lattices. Algebraic geometry and related topics, 169-193.

[257] J. Jost and S.-T. Yau, Harmonic maps and superrigidity. Differential geometry: partial differential equations on manifolds, 245-280, Proc. Sympos. Pure Math., 54, Part 1, Amer. Math. Soc., Providence, RI, 1993.

[258] D. D. Joyce, Compact 8-manifolds with holonomy $\operatorname{Spin}(7)$. Invent. Math. 123(1996), no. $3,507-552$. 
[259] O. D. Joyce, Compact Riemannian 7-manifolds with holonomy $G_{2}$. I, II. J. Differential Geom. 43(1996), no. 2, 291-328, 329-375.

[260] D. Joyce, A new construction of compact 8-manifolds with holonomy Spin(7). J. Differential Geom. 53(1999), no. 1, 89-130.

[261] A. B. Katok, Ergodic properties of degenerate integrable Hamiltonian systems. Izv. Akad. Nauk SSSR. 37(1973) (Russian). Math. USSR-Izv. 7(1973) 535-571.

[262] J. Kim, C. LeBrun and M. Pontecorvo, Scalar-flat Kähler surfaces of all genera. J. Reine Angew. Math. 486(1997), 69-95.

[263] J. R. King, The currents defined by analytic varieties. Acta Math. 127(1971), no. 3-4, $185-220$

[264] S. Klainerman and F. Nicolò, Peeling properties of asymptotically flat solutions to the Einstein vacuum equations". Classical Quantum Gravity 20(2003), no. 14, 3215-3257.

[265] S. Klainerman and S. Selberg, Bilinear estimates and applications to nonlinear wave equations. Commun. Contemp. Math. 4(2002), no. 2, 223-295.

[266] W. Klingenberg, Lectures on closed geodesics. Third edition. Mathematisches Institut der Universität Bonn, Bonn, 1977.

[267] S. Kobayashi, On compact Kähler manifolds with positive definite Ricci tensor. Ann. of Math. (2) 74(1961) 570-574.

[268] K. Kodaira, On a differential-geometric method in the theory of analytic stacks. Proc. Nat. Acad. Sci. U. S. A. 39(1953). 1268-1273.

[269] K. Kodaira, On Kähler varieties of restricted type (an intrinsic characterization of algebraic varieties). Ann. of Math. (2) 60(1954). 28-48.

[270] J. Kollár, Effective base point freeness. Math. Ann. 296(1993), no. 4, 595-605.

[271] M. Kontsevich, Enumeration of rational curves via torus actions. The moduli space of curves (Texel Island, 1994), 335-368, Progr. Math., 129, Birkhäuser, Boston, 1995.

[272] M. Kontsevich, Feynman diagrams and low-dimensional topology. First European Congress of Mathematics, Vol. II (Paris, 1992), 97-121, Progr. Math., 120, Birkhäuser, Basel, 1994

[273] M. Kontsevich, Homological algebra of mirror symmetry. Proceedings of the International Congress of Mathematicians, Vol. 1, 2 (Zürich, 1994), 120-139, Birkhäuser, Basel, 1995.

[274] N. Korevaar, An easy proof of the interior gradient bound for solutions to the prescribed mean curvature equation. Proc. Sympos. Pure Math., 45, Part 2, 81-89, Amer. Math. Soc., Providence, RI, 1986.

[275] N. Korevaar, Upper bounds for eigenvalues of conformal metrics. J. Differential Geom. 37(1993), no. 1, 73-93.

[276] N. Korevaar, R. Mazzeo, F. Pacard, and R. Schoen, Refined asymptotics for constant scalar curvature metrics with isolated singularities. Invent. Math. 135(1999), no. 2, 233272.

[277] N. Korevaar and R. Schoen, Sobolev spaces and harmonic maps for metric space targets. Comm. Anal. Geom. 1(1993), no. 3-4, 561-659

[278] P. Kramer, Group actions on compact hyperbolic manifolds and closed geodesics. Symmetry in physics, 113-124, CRM Proc. Lecture Notes, 34, Amer. Math. Soc., Providence, RI, 2004.

[279] P. B. Kronheimer, and T. S. Mrowka, Embedded surfaces and the structure of Donaldson's polynomial invariants. J. Differential Geom. 41(1995), no. 3, 573-734.

[280] M. Kuranishi, Strongly pseudoconvex CR structures over small balls. I. An a priori estimate. Ann. of Math. (2) 115(1982), no. 3, 451-500.

[281] M. Kuranishi, Strongly pseudoconvex CR structures over small balls. II. A regularity theorem. Ann. of Math. (2) 116(1982), no. 1, 1-64.

[282] M. Kuranishi, Strongly pseudoconvex CR structures over small balls. III. An embedding theorem. Ann. of Math. (2) 116(1982), no. 2, 249-330. 
[283] H. B. Lawson and J. Simons, On stable currents and their application to global problems in real and complex geometry. Ann. of Math. (2) 98(1973), 427-450.

[284] H. B. Lawson Jr. and S.-T. Yau, Compact manifolds of nonpositive curvature. J. Differential Geometry $\mathbf{7}(1972), 211-228$.

[285] H. B. Lawson Jr. and S.-T. Yau, Scalar curvature, non-abelian group actions, and the degree of symmetry of exotic spheres. Comment. Math. Helv. 49(1974), 232-244.

[286] C. R. LeBrun, Explicit Self-Dual Metrics on $\mathbb{C P}_{2} \# \cdots \# \mathbb{C P}_{2}$. J. Diff. Geom. 34(1991), $223-253$

[287] C. LeBrun, Ricci curvature, minimal volumes, and Seiberg-Witten theory. Invent. Math. 145(2001), no. 2, 279-316.

[288] Y. Y. Lee, Embedded special Lagrangian submanifolds in Calabi-Yau manifolds. Comm. Anal. Geom. 11(2003), no. 3, 391-423.

[289] N. C. C. Leung, Einstein type metrics and stability on vector bundles. J. Differential Geom. 45(1997), no. 3, 514-546.

[290] N. C. C. Leung, Lagrangian submanifolds in hyperKähler manifolds, Legendre transformation. J. Differential Geom. 61(2002), no. 1, 107-145.

[291] N. C. C. Leung, Topological quantum field theory for Calabi-Yau threefolds and $G_{2}$ manifolds. Adv. Theor. Math. Phys. 6(2002), no. 3, 575-591.

[292] J. Li, Algebraic geometric interpretation of Donaldson's polynomial invariants. J. Differential Geom. 37(1993), no. 2, 417-466.

[293] J. Li, Stable morphisms to singular schemes and relative stable morphisms. J. Differential Geom. 57(2001), no. 3, 509-578.

[294] J. Li, A degeneration formula of $G W$-invariants. J. Differential Geom. 60(2002), no. 2, 199-293.

[295] J. Li, C. C. M. Liu, K. F. Liu and J. Zhou, A Mathematical Theory of the Topological Vertex Authors, arXiv:math.AG/0408426.

[296] J. Li and G. Tian, Virtual moduli cycles and Gromov-Witten invariants of algebraic varieties. J. Amer. Math. Soc. 11(1998), no. 1, 119-174.

[297] J. Li and G. Tian, Virtual moduli cycles and Gromov-Witten invariants of general symplectic manifolds. Topics in symplectic 4-manifolds, 47-83, First Int. Press Lect. Ser., I, Internat. Press, Cambridge, MA, 1998.

[298] J. Li and S. T. Yau, Hermitian-Yang-Mills connection on non-Kähler manifolds. Mathematical aspects of string theory (San Diego, Calif., 1986), 560-573, Adv. Ser. Math. Phys., 1, World Sci. Publishing, Singapore, 1987.

[299] J. Li and S.-T. Yau, The Existence of Supersymmetric String Theory with Torsion. arXiv:hep-th/0411136.

[300] J. Li, S.-T. Yau and F. Y. Zheng, A simple proof of Bogomolov's theorem on class $\mathrm{VII}_{0}$ surfaces with $b_{2}=0$. Illinois J. Math. 34(1990), no. 2, 217-220.

[301] P. Li, A lower bound for the first eigenvalue of the Laplacian on a compact manifold. Indiana Univ. Math. J. 28(1979), no. 6, 1013-1019.

[302] P. Li, Harmonic sections of polynomial growth. Math. Res. Lett. 4(1997), no. 1, 35-44.

[303] P. Li, R. Schoen and S.-T. Yau, On the isoperimetric inequality for minimal surfaces. Ann. Scuola Norm. Sup. Pisa Cl. Sci. (4) 11(1984), no. 2, 237-244.

[304] P. Li and G. Tian, On the heat kernel of the Bergmann metric on algebraic varieties. J. Amer. Math. Soc. 8(1995), no. 4, 857-877.

[305] P. Li and J. P. Wang, Counting dimensions of L-harmonic functions. Ann. of Math. (2) 152 (2000), no. 2, 645-658.

[306] P. Li and J. P. Wang, Complete manifolds with positive spectrum. II. J. Differential Geom. 62(2002), no. 1, 143-162.

[307] P. Li and S.-T. Yau, Estimates of eigenvalues of a compact Riemannian manifold. Geometry of the Laplace operator, 205-239, Proc. Sympos. Pure Math., XXXVI, Amer. Math. Soc., Providence, R.I., 1980. 
[308] P. Li and S.-T. Yau, A new conformal invariant and its applications to the Willmore conjecture and the first eigenvalue of compact surfaces. Invent. Math. 69(1982), no. 2, 269-291.

[309] P. Li and S.-T. Yau, On the parabolic kernel of the Schrödinger operator. Acta Math. 156(1986), no. 3-4, 153-201.

[310] P. Li and S.-T. Yau, Curvature and holomorphic mappings of complete Kähler manifolds. Compositio Math. 73(1990), no. 2, 125-144.

[311] T. J. Li, Symplectic 4-manifolds with Kodaira dimension zero. Preprint.

[312] T. J. Li and A. Liu, General wall crossing formula. Math. Res. Lett. 2(1995), no. 6, $797-810$

[313] T. J. Li and A. Liu, Symplectic structure on ruled surfaces and a generalized adjunction formula. Math. Res. Lett. 2(1995), no. 4, 453-471.

[314] B. H. Lian, K. F. Liu and S.-T. Yau, Mirror principle. I. Asian J. Math. 1(1997), no. 4 $729-763$.

[315] B. H. Lian, K. F. Liu and S.-T. Yau, Mirror principle. II. Sir Michael Atiyah: a great mathematician of the twentieth century. Asian J. Math. 3(1999), no. 1, 109-146.

[316] B. H. Lian, K. F. Liu and S.-T. Yau, Mirror principle. III. Asian J. Math. 3(1999), no. 4, 771-800.

[317] B. H. Lian, K. F. Liu anf S.-T. Yau, Mirror principle. IV. Surveys in differential geometry, 475-496, Surv. Differ. Geom., VII, Int. Press, Somerville, MA, 2000.

[318] A. Lichnerowicz, Spineurs harmoniques. C. R. Acad. Sci. Paris, 257(1963), 7-9.

[319] A. Lichnerowicz, Magnetohydrodynamics: waves and shock waves in curved space-time. Mathematical Physics Studies, 14. Kluwer Academic Publishers Group, Dordrecht, 1994.

[320] C. S. Lin, The local isometric embedding in $R^{3}$ of 2-dimensional Riemannian manifolds with nonnegative curvature. J. Differential Geometry 21(1985), 213-230.

[321] C. S. Lin, The local isometric embedding in $R^{3}$ of two-dimensional Riemannian manifolds with Gaussian curvature changing sign cleanly. Comm. Pure Appl. Math. 39(1986) 867887.

[322] F. H. Lin, Asymptotic behavior of area-minimizing currents in hyperbolic space. Comm. Pure Appl. Math. 42(1989), no. 3, 229-242.

[323] F. H. Lin, Nodal sets of solutions of elliptic and parabolic equations. Comm. Pure Appl. Math. 44(1991), no. 3, 287-308.

[324] F. H. Lin, Gradient estimates and blow-up analysis for stationary harmonic maps. Ann. of Math. (2) 149(1999), no. 3, 785-829.

[325] F. H. Lin, Mapping problems, fundamental groups and defect measures. Acta Math. Sin. (Engl. Ser.) 15(1999), no. 1, 25-52.

[326] H. Lindblad and I. Rodnianski, Global existence for the Einstein vacuum equations in wave coordinates. Comm. Math. Phys. 256(2005), no. 1, 43-110.

[327] A. K. Liu, Some new applications of general wall crossing formula, Gompf's conjecture and its applications. Math. Res. Lett. 3(1996), no. 5, 569-585.

[328] C. C. M. Liu and S.-T. Yau, Positivity of Quasilocal Mass, Phys. Rev. Lett. 90(2003), no. 23,2311024 pp.

[329] C. C. M. Liu and S.-T. Yau, Positivity of quasi-local mass II. arXiv:math.DG/0412292.

[330] G. Liu and G. Tian, Floer homology and Arnold conjecture. J. Differential Geom. 49(1998), no. 1, 1-74.

[331] K. F. Liu, On modular invariance and rigidity theorems. J. Differential Geom. 41(1995), no. 2, 343-396.

[332] K. F. Liu, On elliptic genera and theta-functions. Topology 35(1996), no. 3, 617-640.

[333] K. F. Liu, X. F. Sun and S.-T. Yau, Canonical metrics on the moduli space of Riemann surfaces. I. J. Differential Geom. 68(2004), no. 3, 571-637.

[334] K. F. Liu, X. F. Sun and S.-T. Yau, Canonical Metrics on the Moduli Space of Riemann Surfaces. II. arXiv.math.DG/0409220. 
[335] J. C. Loftin, Affine spheres and convex $\mathbb{R P}^{n}$-manifolds. Amer. J. Math. 123(2001), no. $2,255-274$.

[336] J. Loftin, S.-T. Yau and E. Zaslow, Affine Manifolds, SYZ Geometry, and the "Y" Vertex Authors. arXiv:math.DG/0405061.

[337] E. Looijenga, $L^{2}$-cohomology of locally symmetric varieties. Compositio Math. 67(1988), no. $1,3-20$.

[338] Z. Q. Lu, On the lower order terms of the asymptotic expansion of Tian-Yau-Zelditch, Amer. J. Math. 122(2000), 235 - 273.

[339] P. Lu and G. Tian, The complex structure on a connected sum of $S^{3} \times S^{3}$ with trivial canonical bundle. Math. Ann. 298(1994), no. 4, 761-764.

[340] A. Lubotzky, R. Phillips and P. Sarnak, Ramanujan graphs. Combinatorica 8(1988), no. $3,261-277$.

[341] H. Luo, Geometric criterion for Gieseker-Mumford stability of polarized manifolds, J. Differential Geom. 49(1998), no. 3, 577-599.

[342] G. Lusztig, Novikov's higher signature and families of elliptic operators. J. Differential Geometry 7(1972), 229-256.

[343] E. Lutwak, Selected affine isoperimetric inequalities. Handbook of convex geometry, Vol. A, B, 151-176, North-Holland, Amsterdam, 1993.

[344] E. Lutwak, D. Yang and G. Y. Zhang, $L_{p}$ affine isoperimetric inequalities. J. Differential Geom. 56(2000), no. 1, 111-132.

[345] T. Mabuchi, K-energy maps integrating Futaki invariants. Tohoku Math. J. (2) 38(1986), no. 4, 575-593.

[346] G.A. Margulis, Discrete groups of motions of manifolds of nonpositive curvature. Transl., I. Ser., Am. Math. Soc. 109(1977), 33-45.

[347] G. A. Margulis, Explicit constructions of graphs without short cycles and low density codes. Combinatorica 2(1982), no. 1, 71-78.

[348] T. Matsusaka, Polarized varieties with a given Hilbert polynomial. Amer. J. Math. 94(1972), 1027-1077.

[349] Y. Matsushima, Sur la structure du group d'homeomorphismes analytiques d'une certaine varietie Kaehleriennes. Nagoya Math. J., 11(1957), 145-150.

[350] Y. Matsushima, On the first Betti number of compact quotient spaces of higherdimensional symmetric spaces. Ann. of Math. (2) 75(1962), 312-330.

[351] D. McDuff, The structure of rational and ruled symplectic 4-manifolds. J. Amer. Math. Soc. 3(1990), no. 3, 679-712.

[352] H. P. McKean, Jr and I. M. Singer, Curvature and the eigenvalues of the Laplacian. J. Differential Geometry 1(1967), no. 1, 43-69.

[353] W. H. Meeks, III and H. Rosenberg, The uniqueness of the helicoid. Ann. of Math. (2) 161(2005), no. 2, 727-758.

[354] W. H. Meeks, L. Simon and S.-T. Yau, Embedded minimal surfaces, exotic spheres, and manifolds with positive Ricci curvature. Ann. of Math. (2) 116(1982), no. 3, 621-659.

[355] W. H. Meeks and S.-T. Yau, Topology of three-dimensional manifolds and the embedding problems in minimal surface theory. Ann. of Math. (2) 112(1980), no. 3, 441-484.

[356] W. H. Meeks and S. T. Yau, The equivariant Dehn's lemma and loop theorem. Comment. Math. Helv. 56(1981), no. 2, 225-239.

[357] W. H. Meeks and S.-T. Yau, The existence of embedded minimal surfaces and the problem of uniqueness. Math. Z. 179(1982), no. 2, 151-168.

[358] W. H. Meeks and S. T. Yau, The classical Plateau problem and the topology of threedimensional manifolds. The embedding of the solution given by Douglas-Morrey and an analytic proof of Dehn's lemma. Topology 21(1982), no. 4, 409-442.

[359] A. d. Melas, On the nodal line of the second eigenfunction of the Laplacian in $R^{2}$. J. Differential Geom. 35(1992), no. 1, 255-263. 
[360] R. Melrose, The inverse spectral problem for planar domains. Instructional Workshop on Analysis and Geometry, Part I (Canberra, 1995), 137-160, Proc. Centre Math. Appl. Austral. Nat. Univ., 34, Austral. Nat. Univ., Canberra, 1996.

[361] M. J. Micallef, Stable minimal surfaces in Euclidean space. J. Differential Geom. 19(1984), no. 1, 57-84.

[362] M. J. Micallef and J. D. Moore, Minimal two-spheres and the topology of manifolds with positive curvature on totally isotropic two-planes. Ann. of Math. (2) 127(1988), no. 1, 199-227.

[363] M. L. Michelsohn, Kähler manifolds with vanishing first Chern class. Seminar on Differential Geometry, pp. 359-361, Ann. of Math. Stud., 102, Princeton Univ. Press, Princeton, N.J., 1982.

[364] M. L. Michelsohn, On the existence of special metrics in complex geometry. Acta Math. 149(1982), no. 3-4, 261-295.

[365] Y. Miyaoka, On the Chern numbers of surfaces of general type. Invent. Math. 42(1977), $225-237$.

[366] N. Mok, Courbure bisectionnelle positive et variétés algébriques affines. C. R. Acad. Sci. Paris Sér. I Math. 296(1983), no. 11, 473-476.

[367] N. Mok, An embedding theorem of complete Kähler manifolds of positive bisectional curvature onto affine algebraic varieties. Bull. Soc. Math. France 112(1984), 197-250.

[368] N. Mok, The uniformization theorem for compact Kähler manifolds of nonnegative holomorphic bisectional curvature. J. Differential Geom. 27(1988), no. 2, 179-214.

[369] N. Mok, Y. T. Siu and S.-T. Yau, The Poincar-Lelong equation on complete Kähler manifolds. Compositio Math. 44(1981), no. 1-3, 183-218.

[370] N. Mok, Y. T. Siu and S. K. Yeung, Geometric superrigidity. Invent. Math. 113(1993), no. $1,57-83$.

[371] N. Mok and S.-T. Yau, Completeness of the Kähler-Einstein metric on bounded domains and the characterization of domains of holomorphy by curvature conditions. Proc. Sympos. Pure Math., 39, pp.41-59, Amer. Math. Soc., Providence, RI, 1983.

[372] N. Mok and J. Q. Zhong, Curvature characterization of compact Hermitian symmetric spaces. J. Differential Geom. 23(1986), no. 1, 15-67.

[373] N. Mok and J. Q. Zhong, Compactifying complete Kähler-Einstein manifolds of finite topological type and bounded curvature. Ann. of Math. (2) 129(1989), no. 3, 427-470.

[374] S. Mori, Projective manifolds with ample tangent bundles. Ann. of Math. (2) 110(1979), no. 3, 593-606.

[375] W. Müller, Analytic torsion and R-torsion of Riemannian manifolds. Adv. in Math. 28(1978), no. 3, 233-305.

[376] G. D. Mostow, Strong rigidity of locally symmetric spaces. Annals of Mathematics Studies, No. 78. Princeton University Press, Princeton, N.J.; University of Tokyo Press, Tokyo, 1973.

[377] C. T. McMullen, The moduli space of Riemann surfaces is K-"ahler hyperbolic. Ann. of Math. (2) 151(2000), no. 1, 327-357.

[378] A. M. Nadel, Multiplier ideal sheaves and Kähler-Einstein metrics of positive scalar curvature. Ann. of Math. (2) 132(1990), no. 3, 549-596.

[379] N. Nadirashvili, Hadamard's and Calabi-Yau's conjectures on negatively curved and minimal surfaces. Invent. Math. 126(1996), no. 3, 457-465.

[380] R. Neel and D. Stroock, Analysis of the cutlocus via the heat kernel. Surveys in Differential Geomtry IX, International Press, 337-350.

[381] L. Ni, Ancient solution to Kahler-Ricci flow. arXiv:math.DG/0502494.

[382] L. Nirenberg, The Weyl and Minkowski problems in differential geometry in the large. Commun. Pure Appl. Math. 6(1953), 337-394.

[383] S. P. Novikov, Multivalued functions and functionals. An analogue of the Morse theory. Dokl. Akad. Nauk SSSR 260(1981), no. 1, 31-35. 
[384] Y.-G. Oh, Floer cohomology of Lagrangian intersections and pseudo-holomorphic disks. III. Arnol'd-Givental conjecture. The Floer memorial volume, 555-573, Progr. Math., 133, Birkhäuser, Basel, 1995.

[385] S. Osher, A level set formulation for the solution of the Dirichlet problem for HamiltonJacobi equations. SIAM J. Math. Anal. 24(1993), no. 5, 1145-1152.

[386] R. Osserman, A proof of the regularity everywhere of the classical solution to Plateau's problem. Ann. of Math. (2) 91(1970), 550-569.

[387] P. Ozsváth and Z. Szabó, The symplectic Thom conjecture. Ann. of Math. (2) 151(2000), no. $1,93-124$.

[388] T. H. Parker and J. G. Wolfson, Pseudo-holomorphic maps and bubble trees. (English. English summary) J. Geom. Anal. 3(1993), no. 1, 63-98.

[389] G. Perelman, The entropy formula for the Ricci flow and its geometric applications. arXiv:math.DG/0211159.

[390] G. Perelman, Ricci flow with surgery on three-manifolds, arXiv:math.DG/0303109.

[391] G. Perelman, inite extinction time for the solutions to the Ricci flow on certain threemanifolds, arXiv:math.DG/0307245.

[392] L. Pestov and G. Uhlmann, The boundary distance function and the Dirichlet-toNeumann map. Math. Res. Lett. 11(2004), no. 2-3, 285-297.

[393] D.H. Phong and J. Sturm, On stability and the convergence of the Kähler-Ricci flow. arXiv:math.DG/0412185

[394] D. H. Phong and J. Sturm, The Monge-Ampère operator and geodesics in the space of Kähler potentials. arXiv:math.DG/0504157.

[395] J. T. Pitts, Existence and regularity of minimal surfaces on Riemannian manifolds. Mathematical Notes, 27. Princeton University Press, Princeton, N.J.; University of Tokyo Press, Tokyo, 1981.

[396] J. T. Pitts and J. H. Rubinstein, Equivariant minimax and minimal surfaces in geometric three-manifolds. Bull. Amer. Math. Soc. (N.S.) 19(1988), no. 1, 303-309.

[397] A. V. Pogorelov, On the question of the existence of a convex surface with a given sum principal radii of curvature. Usp. Mat. Nauk 8(1953), 127-130.

[398] A. V. Pogorelov, On the improper convex affine hyperspheres. Geometriae Dedicata 1(1972), no. 1, 33-46.

[399] A. V. Pogorelov, Existence of a closed convex hypersurface with prescribed curvature. Dokl. Akad. Nauk SSSR 274(1984), no. 1, 28-31.

[400] G. Pólya and G. Szegö, Isoperimetric Inequalities in Mathematical Physics. Annals of Mathematics Studies, no. 27, Princeton University Press, Princeton, N. J., 1951.

[401] J. Qing, and G. Tian, On the uniqueness of the foliation of spheres of constant mean curvature in asymptotically flat 3-manifolds. arXiv:math.DG/0506005

[402] D. Quillen, Superconnections and the Chern character. Topology 24(1985), no. 1, 89-95.

[403] D. Quillen, Cyclic cohomology and algebra extensions. K-Theory 3(1989), no. 3, 205246.

[404] J. Rosenberg, $C^{*}$-algebras, positive scalar curvature and the Novikov conjecture. Publ. I.H.E.S. No. 58(1983), 197-212.

[405] W. D. Ruan, Canonical coordinates and Bergmann metrics. Comm. Anal. Geom. 6(1998), no. 3, 589-631.

[406] D. R. Ray and I. M. Singer, Analytic torsion for complex manifolds. Ann. of Math. (2) 98(1973), 154-177.

[407] M. Reid, The moduli space of 3-folds with $K=0$ may nevertheless be irreducible. Math. Ann. 278(1987), no. 1-4, 329-334.

[408] J. Ross and R. P. Thomas, An obstruction to the existence of constant scalar curvature Kähler metricsmath. arXiv:math.DG/0412518.

[409] J. Ross and R. P. Thomas, A study of the Hilbert-Mumford criterion for the stability of projective varieties. arXiv:math.AG/0412519. 
[410] H. L. Royden, Report on the Teichmller metric. Proc. Nat. Acad. Sci. U.S.A. 65(1970) 497-499.

[411] Y. B. Ruan, Topological sigma model and Donaldson-type invariants in Gromov theory. Duke Math. J. 83(1996), no. 2, 461-500.

[412] Y. B. Ruan and G. Tian, A mathematical theory of quantum cohomology. J. Differential Geom. 42(1995), no. 2, 259-367.

[413] J. Sacks and K. Uhlenbeck, The existence of minimal immersions of 2-spheres. Ann. of Math. (2) 113(1981), no. 1, 1-24.

[414] L. Saper, On the cohomology of locally symmetric spaces and of their compactifications. Current developments in mathematics, 2002, 219-289, Int. Press, Somerville, MA, 2003.

[415] L. Saper and M. Stern, L $L_{2}$-cohomology of arithmetic varieties. Ann. of Math. (2) 132(1990), no. 1, 1-69.

[416] P. Sarnak, Additive number theory and Maass forms. Lecture Notes in Math., 1052 286-309, Springer, Berlin, 1984.

[417] L. Saloff-Coste, Analysis on Riemannian co-compact covers. Surveys in Differential Geometry, IX, 351-385. International Press, Somerville, MA, 02143.

[418] P. Sarnak, Spectra of hyperbolic surfaces. Bull. Amer. Math. Soc. (N.S.) 40(2003), no. 4, 441-478.

[419] R. Schoen, Conformal deformation of a Riemannian metric to constant scalar curvature. J. Differential Geom. 20 (1984), no. 2, 479-495.

[420] R. Schoen, L. Simon and S.-T. Yau, Curvature estimates for minimal hypersurfaces. Acta Math. 134 (1975), no. 3-4, 275-288.

[421] R. Schoen and K. Uhlenbeck, A regularity theory for harmonic maps. J. Differential Geom. 17(1982), no. 2, 307-335.

[422] R. Schoen and K. Uhlenbeck, Boundary regularity and the Dirichlet problem for harmonic maps. J. Differential Geom. 18(1983), no. 2, 253-268.

[423] R. Schoen and J. Wolfson, Minimizing area among Lagrangian surfaces: the mapping problem. J. Differential Geom. 58(2001), no. 1, 1-86.

[424] R. Schoen and S.-T. Yau, On univalent harmonic maps between surfaces. Invent. Math. 44(1978), no. 3, 265-278.

[425] R. Schoen and S. T. Yau, Existence of incompressible minimal surfaces and the topology of three-dimensional manifolds with nonnegative scalar curvature. Ann. of Math. (2) 110(1979), no. 1, 127-142.

[426] R. Schoen and S.-T. Yau, On the proof of the positive mass conjecture in general relativity. Comm. Math. Phys. 65(1979), no. 1, 45-76.

[427] R. Schoen and S.-T. Yau, Positivity of the total mass of a general space-time. Phys. Rev. Lett. 43(1979), no. 20, 1457-1459.

[428] R. Schoen and S.-T. Yau, On the structure of manifolds with positive scalar curvature. Manuscripta Math. 28(1979), no. 1-3, 159-183.

[429] R. Schoen and S.-T. Yau, Compact group actions and the topology of manifolds with nonpositive curvature. Topology 18(1979), no. 4, 361-380.

[430] R. Schoen and S.-T. Yau, Proof of the positive mass theorem. II. Comm. Math. Phys. 79(1981), no. 2, 231-260.

[431] R. Schoen and S.-T. Yau, Complete three-dimensional manifolds with positive Ricci curvature and scalar curvature. Seminar on Differential Geometry, pp. 209-228, Ann. of Math. Stud., 102, Princeton Univ. Press, Princeton, N.J., 1982.

[432] R. Schoen and S.-T. Yau, The existence of a black hole due to condensation of matter. Comm. Math. Phys. 90(1983), no. 4, 575-579.

[433] R. Schoen and S.-T. Yau, Conformally flat manifolds, Kleinian groups and scalar curvature. Invent. Math. 92(1988), no. 1, 47-71.

[434] R. Schoen and S.-T. Yau, Lectures on differential geometry. Lecture notes prepared by Wei Yue Ding, Kung Ching Chang [Gong Qing Zhang], Jia Qing Zhong and Yi Chao $\mathrm{Xu}$. Translated from the Chinese by Ding and S. Y. Cheng. Preface translated from the 
Chinese by Kaising Tso. Conference Proceedings and Lecture Notes in Geometry and Topology, I. International Press, Cambridge, MA, 1994.

[435] N. Seiberg and E. Witten, Electric-magnetic duality, monopole condensation, and confinement in $N=2$ supersymmetric Yang-Mills theory. Nuclear Phys. B 426(1994), no. $1,19-52$.

[436] N. Seiberg and E. Witten, Monopoles, duality and chiral symmetry breaking in $N=2$ supersymmetric QCD. Nuclear Phys. B 431(1994), no. 3, 484-550.

[437] P. Seidel and R. Thomas, Braid group actions on derived categories of coherent sheaves. Duke Math. J. 108(2001), no. 1, 37-108.

[438] J. Shatah and M. Struwe, Regularity results for nonlinear wave equations. Ann. of Math. (2) 138(1993), no. 3, 503-518.

[439] W. X. Shi, Complete noncompact Kähler manifolds with positive holomorphic bisectional curvature. Bull. Amer. Math. Soc. (N.S.) 23(1990), no. 2, 437-440.

[440] W.-X. Shi, Ricci flow and the uniformization on complete noncompact Kähler manifolds. J. Differential Geom. 45(1997), no. 1, 94-220.

[441] W. X. Shi, A uniformization theorem for complete Kähler manifolds with positive holomorphic bisectional curvature. J. Geom. Anal. 8(1998), no. 1, 117-142.

[442] Y. G. Shi and L. F. Tam, Positive mass theorem and the boundary behaviors of compact manifolds with nonnegative scalar curvature. J. Differential Geom. 62(2002), no. 1, 79125.

[443] B. Shiffman, Complete characterization of holomorphic chains of codimension one. Math. Ann. 274(1986), no. 2, 233-256.

[444] L. Simon, Theorems on the regularity and singularity of minimal surfaces and harmonic maps. Geometry and global analysis (Sendai, 1993), 111-145,

[445] L. Simon, Existence of Willmore surfaces. Miniconference on geometry and partial differential equations (Canberra, 1985), 187-216, Proc. Centre Math. Anal. Austral. Nat. Univ., 10, Austral. Nat. Univ., Canberra, 1986.

[446] L. Simon, Theorems on regularity and singularity of energy minimizing maps. Lectures in Mathematics ETH Zürich. Birkhäuser Verlag, Basel, 1996.

[447] C. T. Simpson, Constructing variations of Hodge structure using Yang-Mills theory and applications to uniformization. J. Amer. Math. Soc. 1(1988), no. 4, 867-918.

[448] C. T. Simpson, Moduli of representations of the fundamental group of a smooth projective variet. I. Inst. Hautes Études Sci. Publ. Math. No. 79(1994), 47-129.

[449] C. T. Simpson, Moduli of representations of the fundamental group of a smooth projective variety. II. Inst. Hautes tudes Sci. Publ. Math. No. 80(1994), 5-79 (1995).

[450] I. M. Singer, B. Wong, S.-T. Yau and S.-T. S. Yau, An estimate of the gap of the first two eigenvalues in the Schrödinger operator. Ann. Scuola Norm. Sup. Pisa Cl. Sci. (4) 12(1985), no. 2, 319-333.

[451] Y.-T. Siu, Complex-analyticity of harmonic maps and strong rigidity of compact Kähler manifolds. Ann. of Math. 112(1980), 73-111.

[452] Y. T. Siu, A simple proof of the surjectivity of the period map of K3 surfaces. Manuscripta Math. 35(1981), no. 3, 311-321.

[453] Y. T. Siu, Every K3 surface is Kähler. Invent. Math. 73(1983), no. 1, 139-150.

[454] Y. T. Siu, Effective very ampleness. Invent. Math. 124(1996), no. 1-3, 563-571.

[455] Y. T. Siu, Invariance of plurigenera. Invent. Math. 134(1998), no. 3, 661-673.

[456] Y. T. Siu, Very ampleness part of Fujita's conjecture and multiplier ideal sheaves of Kohn and Nadel. Complex analysis and geometry (Columbus, OH, 1999), 171-191, Ohio State Univ. Math. Res. Inst. Publ., 9, de Gruyter, Berlin, 2001.

[457] Y. T. Siu and S.-T. Yau, Complete Kähler manifolds with nonpositive curvature of faster than quadratic decay. Ann. of Math. (2) 105(1977), no. 2, 225-264.

[458] Y. T. Siu and S.-T. Yau, Compact Kähler manifolds of positive bisectional curvature. Invent. Math. 59(1980), no. 2, 189-204. 
[459] Y. T. Siu and S.-T. Yau, Compactification of negatively curved complete Kähler manifolds of finite volume. Seminar on Differential Geometry, pp. 363-380, Ann. of Math. Stud., 102, Princeton Univ. Press, Princeton, N.J., 1982.

[460] I. Smith, R. P. Thomas and S.-T. Yau, Symplectic conifold transitions. J. Differential Geom. 62(2002), no. 2, 209-242.

[461] K. Smoczyk, Longtime existence of the Lagrangian mean curvature flow. Calc. Var. Partial Differential Equations 20(2004), no. 1, 25-46.

[462] K. Smoczyk and M. T. Wang, Mean curvature flows of Lagrangians submanifolds with convex potentials. J. Differential Geom. 62(2002), no. 2, 243-257.

[463] J. A. Smoller, A. G. Wasserman and S.-T. Yau, Existence of black hole solutions for the Einstein-Yang/Mills equations. Comm. Math. Phys. 154(1993), no. 2, 377-401.

[464] J. A. Smoller, A. G. Wasserman, S.-T. Yau, J. B. McLeod, Smooth static solutions of the Einstein/Yang-Mills equations. Comm. Math. Phys. 143(1991), no. 1, 115-147.

[465] M. Stern, $L^{2}$-index theorems on locally symmetric spaces. Invent. Math. 96(1989), no. 2, 231-282.

[466] S. Stolz, Simply connected manifolds of positive scalar curvature. Ann. of Math. (2) 136(1992), no. 3, 511-540.

[467] A. Strominger, Superstrings with torsion. Nuclear Phys. B 274(1986), no. 2, 253-284.

[468] A. Strominger, S.-T. Yau and E. Zaslow, Mirror symmetry is T-duality. Nuclear Phys. B 479(1996), no. 1-2, 243-259.

[469] D. W. Stroock, An introduction to the analysis of paths on a Riemannian manifold. Mathematical Surveys and Monographs, 74. American Mathematical Society, Providence, RI, 2000.

[470] D. Sullivan, A generalization of Milnor's inequality concerning affine foliations and affine manifolds. Comment. Math. Helv. 51(1976), no. 2, 183-189.

[471] D. Sullivan, The Dirichlet problem at infinity for a negatively curved manifold. J. Differential Geom. 18(1983), no. 4, 723-732.

[472] G. Szegö, G. Inequalities for certain eigenvalues of a membrane of given area. J. Rational Mech. Anal. 3(1954), 343-356.

[473] G. Talenti, Best constant in Sobolev inequality. Ann. Mat. Pura Appl. (4) 110(1976), $353-372$

[474] T. Tao, Low-regularity global solutions to nonlinear dispersive equations. Surveys in analysis and operator theory (Canberra, 2001), 19-48, Proc. Centre Math. Appl. Austral. Nat. Univ., 40, Austral. Nat. Univ., Canberra, 2002.

[475] D. Tataru, On global existence and scattering for the wave maps equation. Amer. J. Math. 123(2001), no. 1, 37-77.

[476] C. H. Taubes, Self-dual Yang-Mills connections on non-self-dual 4-manifolds. J. Differential Geom. 17(1982), no. 1, 139-170.

[477] C. H. Taubes, Self-dual connections on 4-manifolds with indefinite intersection matrix. J. Differential Geom. 19(1984), no. 2, 517-560.

[478] C. H. Taubes, $S^{1}$ actions and elliptic genera. Comm. Math. Phys. 122(1989), no. 3, $455-526$

[479] C. H. Taubes, Casson's invariant and gauge theory. J. Differential Geom. 31(1990), no. $2,547-599$.

[480] C. H. Taubes, The existence of anti-self-dual conformal structures. J. Differential Geom. 36(1992), no. 1, 163-253.

[481] C. H. Taubes, The Seiberg-Witten invariants and symplectic forms. Math. Res. Lett. 1(1994), no. 6, 809-822.

[482] H. C. Taubes, SW $\Rightarrow$ Gr: from the Seiberg-Witten equations to pseudo-holomorphic curves. J. Amer. Math. Soc. 9(1996), no. 3, 845-918.

[483] C. H. Taubes, Counting pseudo-holomorphic submanifolds in dimension 4. J. Differential Geom. 44(1996), no. 4, 818-893. 
[484] C. H. Taubes, Gr $\Longrightarrow \mathrm{SW}$ : from pseudo-holomorphic curves to Seiberg-Witten solutions. J. Differential Geom. 51(1999), no. 2, 203-334.

[485] C. H. Taubes, GR = SW: counting curves and connections. J. Differential Geom. 52(1999), no. 3, 453-609.

[486] C. H. Taubes, Seiberg Witten and Gromov invariants for symplectic 4-manifolds. Edited by Richard Wentworth. First International Press Lecture Series, 2. International Press, Somerville, MA, 2000.

[487] C. L. Terng, A higher dimension generalization of the sine-Gordon equation and its soliton theory. Ann. of Math. (2)111(1980), no. 3, 491-510.

[488] C. L. Terng, Recent progress in submanifold geometry. Differential geometry: partial differential equations on manifolds, 439-484, Proc. Sympos. Pure Math., 54, Part 1, Amer. Math. Soc., Providence, RI, 1993.

[489] C. L. Terng, Soliton equations and differential geometry. J. Differential Geom. 45(1997), no. $2,407-445$.

[490] C. L. Terng and K. Uhlenbeck, Poisson actions and scattering theory for integrable systems. Surv. Differ. Geom., V, 315-402. Int. Press, Boston, MA, 1998.

[491] C. L. Terng and K. Uhlenbeck, Bäcklund transformations and loop group actions. Comm. Pure Appl. Math. 53 (2000), no. 1, 1-75.

[492] R.P. Thomas and S.-T. Yau, Special Lagrangians, stable bundles and mean curvature flow. (English. English summary) Comm. Anal. Geom. 10(2002), no. 5, 1075-1113.

[493] G. Thorbergsson, A survey on isoparametric hypersurfaces and their generalizations. Handbook of differential geometry, Vol. I, 963-995, North-Holland, Amsterdam, 2000.

[494] W. P. Thurston, Three-dimensional manifolds, Kleinian groups and hyperbolic geometry. Bull. Amer. Math. Soc. (N.S.) 6(1982), no. 3, 357-381.

[495] W. Thurston, Three-dimensional Geometry and Topology, Vol. 1 (Silvio Levy, ed.) Princeton Math. Ser., vol. 35, Princeton Univ. Press, 1997.

[496] G. Tian, Smoothness of the universal deformation space of compact Calabi-Yau manifolds and its Petersson-Weil metric. Mathematical aspects of string theory (San Diego, Calif., 1986), 629-646, Adv. Ser. Math. Phys., 1, World Sci. Publishing, Singapore, 1987.

[497] G. Tian, On a set of polarized Kähler metrics on algebraic manifolds. J. Differential Geom. 32(1990), no. 1, 99-130.

[498] G. Tian, Calabi's conjecture for complex surfaces with positive first Chern class. Invent. Math. 101(1990), no. 1, 101-172.

[499] G. Tian, Kähler-Einstein metrics with positive scalar curvature. Invent. Math. 130(1997), no. 1, 1-37.

[500] G. Tian, Gauge theory and calibrated geometry. I. Ann. of Math. (2) 151(2000), no. 1, 193-268.

[501] G. Tian and S.-T. Yau, Three-dimensional algebraic manifolds with $C_{1}=0$ and $\chi=-6$. Mathematical aspects of string theory (San Diego, Calif., 1986), 543-559, Adv. Ser. Math. Phys., 1, World Sci. Publishing, Singapore, 1987.

[502] G. Tian and S.-T. Yau, Complete Kähler manifolds with zero Ricci curvature. I. J. Amer. Math. Soc. 3(1990), no. 3, 579-609.

[503] G. Tian and S.-T. Yau, Complete Kähler manifolds with zero Ricci curvature. II. Invent. Math. 106(1991), no. 1, 27-60.

[504] A. N. Todorov, Applications of the Kähler-Einstein-Calabi-Yau metric to moduli of K3 surfaces. Invent. Math. 61(1980), no. 3, 251-265.

[505] A. N. Todorov, The Weil-Petersson geometry of the moduli space of $\mathrm{SU}(n \geq 3)$ (CalabiYau) manifolds. I. Comm. Math. Phys. 126(1989), no. 2, 325-346.

[506] A. E. Treibergs, Entire spacelike hypersurfaces of constant mean curvature in Minkowski space. Invent. Math. 66(1982), no. 1, 39-56.

[507] N. S. Trudinger, Remarks concerning the conformal deformation of Riemannian structures on compact manifolds. Ann. Scuola Norm. Sup. Pisa (3) 22(1968), 265-274. 
[508] N. S. Trudinger and X. J. Wang, The Bernstein problem for affine maximal hypersurfaces. Invent. Math. 140(2000), no. 2, 399-422.

[509] K. K. Uhlenbeck, Removable singularities in Yang-Mills fields. Comm. Math. Phys. 83(1982), no. 1, 11-29

[510] K. K. Uhlenbeck, Connections with $L^{p}$ bounds on curvature. Comm. Math. Phys. 83(1982), no. 1, 31-42.

[511] K. Uhlenbeck, Harmonic maps into Lie groups: classical solutions of the chiral model. J. Differential Geom. 30(1989), no. 1, 1-50.

[512] K. Uhlenbeck, and S.-T. Yau, On the existence of Hermitian-Yang-Mills connections in stable vector bundles. Comm. Pure Appl. Math. 39(1986), no. S, suppl., S257-S293.

[513] C. Vafa, Topological mirrors and quantum rings. Essays on mirror manifolds, 96-119, Internat. Press, Hong Kong, 1992.

[514] C. Verdiere, Theoremes de Courant et de Cheng combinatoires. Combinatorial Courant and Cheng theorems Seminaire de Theorie Spectrale et Geometrie, No. 13, Annee 19941995, 9-13.

[515] T. Wan, Constant mean curvature surface, harmonic maps, and universal Teichmüller space. J. Differential Geom. 35(1992), no. 3, 643-657.

[516] C. P. Wang, Some examples of complete hyperbolic affine 2-spheres in $R^{3}$. Global differential geometry and global analysis (Berlin, 1990), 271-280, Lecture Notes in Math. 1481, Springer, Berlin, 1991.

[517] M. T. Wang, A fixed point theorem of discrete group actions on Riemannian manifolds. J. Differential Geom. 50(1998), no. 2, 249-267.

[518] M. T. Wang, Generalized harmonic maps and representations of discrete groups. Comm. Anal. Geom. 8(2000), no. 3, 545-563.

[519] M. T. Wang, Mean curvature flow of surfaces in Einstein four-manifolds. J. Differential Geom. 57(2001), no. 2, 301-338.

[520] M. T. Wang, Deforming area preserving diffeomorphism of surfaces by mean curvature flow. Math. Res. Lett. 8(2001), no. 5-6, 651-661.

[521] M. Y. Wang and W. Ziller, Existence and nonexistence of homogeneous Einstein metrics. Invent. Math. 84(1986), no. 1, 177-194.

[522] X. J. Wang and X. H. Zhu, Kähler-Ricci solitons on toric manifolds with positive first Chern class. Adv. Math. 188(2004), no. 1, 87-103.

[523] X. W. Wang, Balance point and stability of vector bundles over a projective manifold. Math. Res. Lett. 9(2002), no. 2-3, 393-411.

[524] S. Weinberger, Aspects of the Novikov conjecture, in Geometric and Topological Invariants of Elliptic Operators. J. Kaminker, editor, (1990), 281-297, A.M.S., Providence, R.I.

[525] N. Wickramasekera, A rigidity theorem for stable minimal hypercones. J. Differential Geom. 68(2004), no. 3, 433-514.

[526] N. Wickramasekera, On the singularities and a Bernstein property of immersed stable minimal hypersurfaces. Calc. Var. Partial Differential Equations 22(2005), no. 1, 1-20.

[527] E. Witten, A new proof of the positive energy theorem. Comm. Math. Phys. 80(1981), no. $3,381-402$.

[528] E. Witten, Supersymmetry and Morse theory J. Differential Geom. 17(1982), no. 4, 661-692 (1983)

[529] E. Witten, Holomorphic Morse inequalities. Algebraic and differential topology-global differential geometry, 318-333, Teubner-Texte Math., 70, Teubner, Leipzig, 1984.

[530] E. Witten, Elliptic genera and quantum field theory. Comm. Math. Phys. 109(1987), no. $4,525-536$.

[531] E. Witten, $2+1$-dimensional gravity as an exactly soluble system. Nuclear Phys. B 311(1988/89), no. 1, 46-78.

[532] E. Witten, On the structure of the topological phase of two-dimensional gravity. Nuclear Phys. B 340(1990), no. 2-3, 281-332. 
[533] E. Witten, Monopoles and four-manifolds. Math. Res. Lett. 1(1994), no. 6, 769-796.

[534] E. Witten and S.-T. Yau, Connectedness of the boundary in the AdS/CFT correspondence. Adv. Theor. Math. Phys. 3(1999), no. 6, 1635-1655 (2000).

[535] M. Wolf, The Teichmuller theory of harmonic maps. J. Differential Geom. 29(1989), no. 2, 449-479.

[536] S. A. Wolpert, Geometry of the Weil-Petersson completion of Teichmüller space. Surveys in differential geometry, Vol. VIII, 357-393. Int. Press, Somerville, MA, 2003.

[537] P. C. Yang and S.-T. Yau, Eigenvalues of the Laplacian of compact Riemann surfaces and minimal submanifolds. Ann. Scuola Norm. Sup. Pisa Cl. Sci. (4) 7(1980), no. 1, $55-63$.

[538] S.-T. Yau, On the fundamental group of compact manifolds of non-positive curvature. Ann. of Math. (2) 93(1971), 579-585.

[539] S.-T. Yau, Harmonic functions on complete Riemannian manifolds. Comm. Pure Appl. Math. 28(1975), 201-228.

[540] S.-T. Yau, Isoperimetric constants and the first eigenvalue of a compact Riemannian manifold. Ann. Sci. cole Norm. Sup. (4) 8(1975), no. 4, 487-507.

[541] S.-T. Yau, Some function-theoretic properties of complete Riemannian manifold and their applications to geometry. Indiana Univ. Math. J. 25(1976), no. 7, 659-670.

[542] S.-T. Yau, Calabi's conjecture and some new results in algebraic geometry. Proc. Nat. Acad. Sci. U.S.A. 74(1977), no. 5, 1798-1799.

[543] S.-T. Yau, On the Ricci curvature of a compact Kähler manifold and the complex MongeAmpère equation. I. Comm. Pure Appl. Math. 31(1978), no. 3, 339-411.

[544] S.-T. Yau, The role of partial differential equations in differential geometry. Proceedings of the International Congress of Mathematicians (Helsinki, 1978), pp. 237-250, Acad. Sci. Fennica, Helsinki, 1980.

[545] S.-T. Yau, A general Schwarz lemma for Kähler manifolds. Amer. J. Math. 100(1978), no. 1, 197-203.

[546] S.-T. Yau, Problem section. Seminar on Differential Geometry, pp. 669-706, Ann. of Math. Stud., 102, Princeton Univ. Press, Princeton, N.J., 1982.

[547] S.-T. Yau, Compact three-dimensional K ähler manifolds with zero Ricci curvature. Symposium on anomalies, geometry, topology (Chicago, Ill., 1985), 395-406, World Sci. Publishing, Singapore, 1985.

[548] S.-T. Yau, Nonlinear analysis in geometry. Monographies de L'Enseignement Mathmatique, 33. Série des Conférences de l'Union Mathématique Internationale, 8. L'Enseignement Mathématique, Geneva, 1986. 54 pp.

[549] S.-T. Yau, A review of complex differential geometry. Proc. Symp. Pur Math. 52(1991), 619-625.

[550] S.-T. Yau, Open problems in Geometry. Proc. Symp. Pure Math. 54(1993) 1-28.

[551] S.-T. Yau, An application of eigenvalue estimate to algebraic curves defined by congruence subgroups. Math. Res. Lett. 3(1996), no. 2, 167-172.

[552] S.-T. Yau, A note on the distribution of critical points of eigenfunctions. Collection of papers on geometry, analysis and mathematical physics, 173-175, World Sci. Publishing, River Edge, NJ, 1997.

[553] S.-T. Yau, An estimate of the gap of the first two eigenvalues in the Schrödinger operator. Lectures on partial differential equations, 223-235, New Stud. Adv. Math., 2, Int. Press, Somerville, MA, 2003.

[554] S.-T. Yau and W. H. Meeks, The equivariant loop theorem for three-dimensional manifolds and a review of the existence theorems for minimal surfaces. The Smith conjecture (New York, 1979), 153-163, Pure Appl. Math., 112, Academic Press, Orlando, FL, 1984.

[555] R. G. Ye, Gromov's compactness theorem for pseudo holomorphic curves. Trans. Amer. Math. Soc. 342(1994), no. 2, 671-694. 
[556] R. G. Ye, Foliation by constant mean curvature spheres on asymptotically flat manifolds. Geometric analysis and the calculus of variations, 369-383, Internat. Press, Cambridge, MA, 1996.

[557] G. L. Yu, Novikov conjecture for groups with finite asymptotic dimension. Ann. of Math. (2) 147(1998), no. 2, 325-355.

[558] S. Zelditch, Szegö kernels and a theorem of Tian. Int. Math. Res. Not. 6(1998), 317-331.

[559] S. Zelditch, Spectral determination of analytic bi-axisymmetric plane domains. Geom. Funct. Anal. 10(2000), no. 3, 628-677.

[560] S. W. Zhang, Heights and reductions of semi-stable varieties. Compositio Math. 104(1996), no. 1, 77-105.

[561] X. Zhang, A definition of total energy-momenta and the positive mass theorem on asymptotically hyperbolic 3-manifolds. I. Comm. Math. Phys. 249(2004), no. 3, 529-548.

[562] J. Q. Zhong and H. C. Yang, On the estimate of the first eigenvalue of a compact Riemannian manifold. Sci. Sinica Ser. A 27(1984), no. 12, 1265-1273.

[563] A. Zinger, Enumeration of genus-two curves with a fixed complex structure in $\mathbb{P}^{2}$ and $\mathbb{P}^{3}$, arXiv:math.SG/0201254.

[564] A. Zinger, Enumerative vs. Invariants and obstruction bundles, arXiv:math.SG/0201255. 